(8) MSNWW

$8551154^{\text {th }}$ Avenue NE

Redmond, WA 98052

\title{
Macron Formed Liner Compression as a Practical Method for Enabling Magneto- Inertial Fusion
}

DOE Award Number:

Recipient Institution:

Street Address/City/State/Zip:

Principal Investigator:

Postal Address:

Telephone Number:

Email:
DE-SC0001224

MSNW LLC

$8551154^{\text {th }}$ Avenue NE

Redmond, WA 98052

Dr. John Slough

$8551154^{\text {th }}$ Ave NE

Redmond, WA 98052

(425) 867-8900

slough@msnwllc.com 


\section{Executive Summary}

The entry of fusion as a viable, competitive source of power has been stymied by the challenge of finding an economical way to provide for the confinement and heating of the plasma fuel. The main impediment for current nuclear fusion concepts is the complexity and large mass associated with the confinement systems. To take advantage of the smaller scale, higher density regime of magnetic fusion, an efficient method for achieving the compressional heating required to reach fusion gain conditions must be found. The very compact, high energy density plasmoid commonly referred to as a Field Reversed Configuration (FRC) provides for an ideal target for this purpose. To make fusion with the FRC practical, an efficient method for repetitively compressing the FRC to fusion gain conditions is required. A novel approach to be explored in this endeavor is to remotely launch a converging array of small macro-particles (macrons) that merge and form a more massive liner inside the reactor which then radially compresses and heats the FRC plasmoid to fusion conditions. The closed magnetic field in the target FRC plasmoid suppresses the thermal transport to the confining liner significantly lowering the imploding power needed to compress the target. With the momentum flux being delivered by an assemblage of low mass, but high velocity macrons, many of the difficulties encountered with the liner implosion power technology are eliminated.

The undertaking to be described in this report was to evaluate the feasibility achieving fusion conditions from this simple and low cost approach to fusion. During phase I the design and testing of the key components for the creation of the macron formed liner have been successfully carried out. Detailed numerical calculations of the merging, formation and radial implosion of the Macron Formed Liner (MFL) were also performed. The phase II effort will focus on an experimental demonstration of the macron launcher at full power, and the demonstration of megagauss magnetic field compression by a small array of full scale macrons. In addition the physics of the compression of an FRC to fusion conditions will be undertaken with a smaller scale MFL. The timescale for testing will be rapidly accelerated by taking advantage of other facilities at MSNW where the target FRC will be created and translated inside the MFL just prior to implosion of the MFL.

Experimental success would establish the concept at the "proof of principle" level and the following phase III effort would focus on the full development of the concept into a fusion gain device. Successful operation would lead to several benefits in various fields. It would have application to high energy density physics, as well as nuclear waste transmutation and alternate fission fuel cycles. The smaller scale device could find immediate application as an intense source of neutrons for diagnostic imaging and non-invasive object interrogation. 


\section{Table of Contents}

Executive Summary 1

Table of Contents 2

I. Significance of the Problem and Technical Approach 3

A. Macroparticle (macron) formed liner concept. 3

B. The Macron Launcher 5

C. Magneto-Inertial Fusion Scaling $\quad 7$

D. Macron Liner Composition $\quad 8$

E. Target FRC Physics $\quad 9$

II. Anticipated Public Benefits 11

III. Degree to which Phase I has demonstrated Technical Feasibility 13

A. Numerical Modeling Results 13

B. Experimental Results 15

C. Macron Launcher Experiments 19

IV. Technical Objectives for phase II 21

V. Phase II Work Plan 23

VI. The Performance Schedule 38

VII. Facilities/Equipment 40

VIII. Bibliography and References 41

Appendix I Journal of Fusion Paper 42 


\section{Significance of the Problem and Technical Approach}

In the pursuit of fusion the major research endeavors have coalesced into exploring two regions that represent the extrema of plasma energy density. Steady state toroidal devices such as the tokamak are found at the low end, and inertial confinement fusion at the high end. Both enterprises will soon reach a condition where more energy is produced by fusion than consumed in creating the fusion plasma. While this will be an impressive achievement, the systems required to achieve these results are equally impressive in size and cost as the cost of development seems invariably linked to system size. For MFE, size reduction is limited by the maximum magnetic field strength obtainable with superconducting magnets. The critical constraint with ICF is the costly high-power drivers needed to achieve the extreme conditions of density and pressure. Another promising approach that could dramatically reduce the system size is the pulse compression of a high beta, magnetically insulated plasma such as the Field Reversed Configuration (FRC) [1]. One method for achieving fusion conditions at high energy density is to employ the kinetic energy of a metal liner to compress the target plasmoid to high density and temperature. The energy density of these systems is intermediate between MFE and ICF, and potentially a better match for efficient power conversion. For liner based systems, the achievement of fusion gain is a hybrid of both MFE and ICF in that the presence of magnetic field in the target plasma suppresses the thermal transport to the confining shell, thus lowering the imploding power needed to compress the target to fusion conditions. Unlike MFE, the confinement time is not determined by the energy confinement of the magnetized plasma, but instead by the liner dwell time determined by the liner inertia. This area of fusion research has thus been dubbed magneto-inertial fusion, or MIF.

To take advantage of the smaller scale, higher density regime of MIF an efficient method for achieving the compressional heating required to reach fusion gain conditions must be found. The method needs to be simple and capable of repetitive operation. The macroparticle (macron) formed liner compression of the FRC is such a method, and uniquely addresses the major challenges facing magneto-inertial fusion. Namely,

(1) It embodies a compression scheme that can very efficiently and repetitively generate the kinetic liner energy required to reach fusion gain.

(2) It provides for both the target plasma and liner energy to be generated remote from the reactor vessel. This is critical as the reactor environment is likely to be incompatible with the specialized pulse power equipment employed in conventional liner approaches.

(3) The timescale for forming and accelerating both the FRC and liner can be much longer than the time that the energy is thermalized in the implosion. This avoids the need for the very high voltages required to produce multi-Megampere compression currents. In fact the switching requirement for the Macron Formed Liner (MFL) is well within the range of currently available solid state devices.

(4) The time and cost for testing the concept is negligible when compared to alternative approaches. The required target FRC plasma is already routinely produced at the MSNW laboratory. The macron liner formation could be tested in phase II with a successful outcome with the prototype macron launcher testing to be performed during phase I. 


\section{A. Macroparticle (macron) formed liner concept.}

The proposed method by which MIF liner fusion can be made practical is quite simple. The key to achieving a workable pulsed fusion reactor is achieving the required standoff for the liner driver and is illustrated in Figs. 1 and 2. The target plasma is assembled by producing two FRCs remote from the burn chamber, and translating them into the center where they merge and form a stable target FRC. The merged FRC must have a decay time long compared to both the FRC assembly and compression time. This aspect of the concept - the FRC formation, acceleration and merging process - has already been successfully demonstrated in the Inductive Plasma Accelerator (IPA) experiments at MSNW [2]. In the reactor application a small $(\mathrm{kG})$ guide field maintains the FRC against radial expansion as illustrated in Fig. 1.

The metallic liner is also produced by merging as well, however in this case by a cluster of small masses (macrons) that are injected into the reactor by an array of launchers, also situated outside the reactor vessel. In this way the energy required to compress the FRC can be (1) delivered on a timescale that is much slower than the FRC compression and (2) produced by a source that is highly efficient, well distributed, and low tech. Most importantly (3) the liner formation system can be located outside the reactor in a manner compatible with long-term repetitive operation.

The macron launchers are initiated in such a manner that a large array of small metallic masses arrive at the central section of the reactor (See Fig. 2) and converge to form a contiguous liner at smaller radius $\left(\mathrm{r}_{\mathrm{L}} \sim 0.1 \mathrm{~m}\right)$. The $\mathrm{FRC}$ is then introduced within the liner by

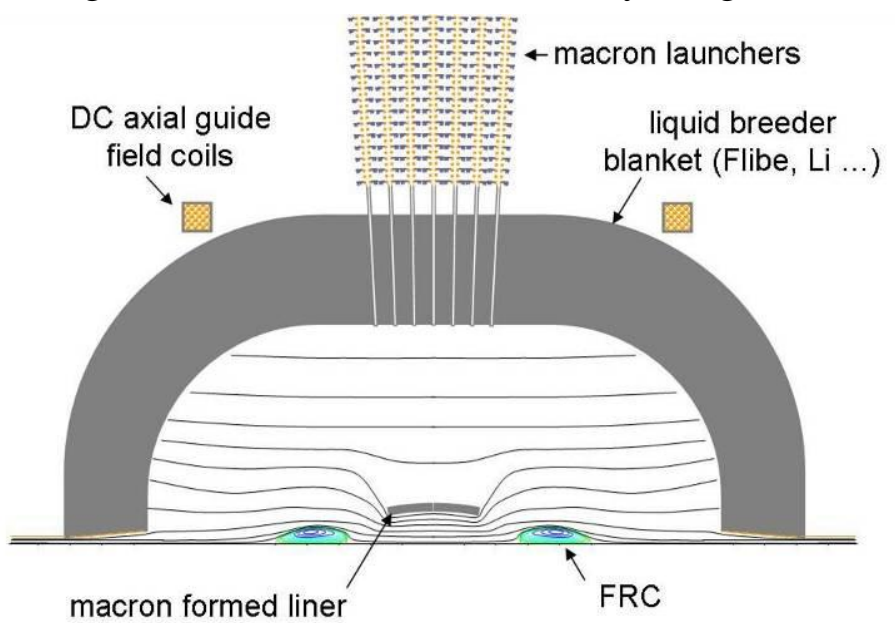

Figure 1. Reactor illustration based on the macron formed liner (MFL) compression of the FRC (R-Z plane). Scale of the FRC and liner has been enlarged for purpose of illustration.

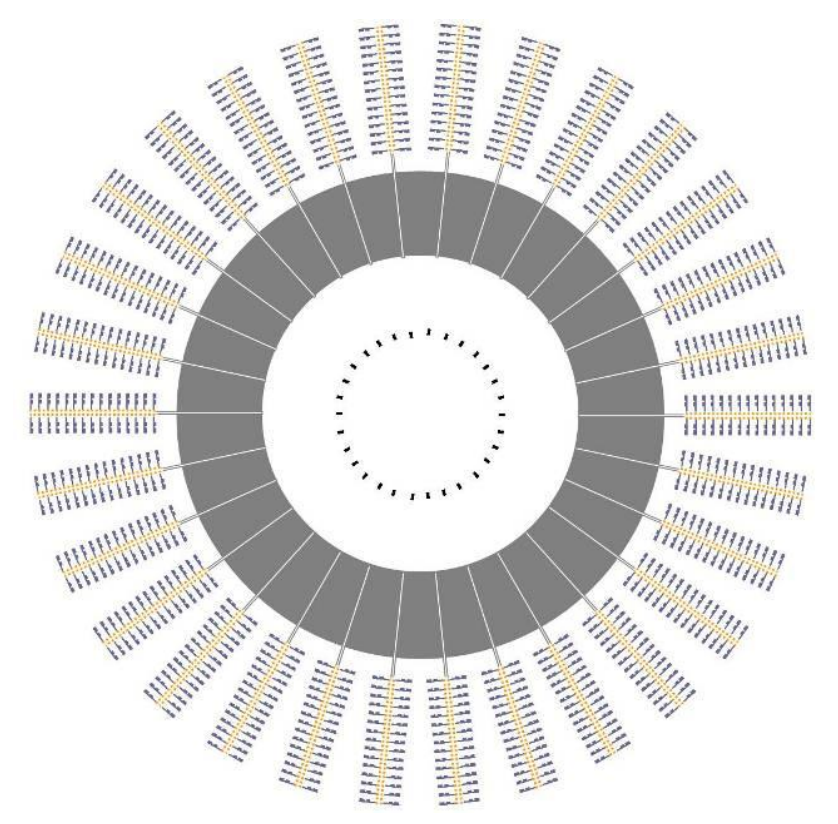

Figure 2. Midplane cross sectional view of reactor based MFL. The macron size was increased and reactor scale reduced for illustration. 
merging two translated FRCs. The macron liner continues to converge, both radially and axially, compressing the FRC to fusion gain conditions.

The approach is similar to that suggested by Thio [3] where the liner formation is based on plasma jet compression. The employment of a macron rather than a plasma jet overcomes the main difficulties with the plasma approach, specifically, achieving sufficient liner inertia and avoiding liner compressibility issues. As with the plasma jets, the use of a directed energy source allows for the liner to have a wide range of properties that are difficult if not impossible to achieve with conventional liners such as axial compression and rotation. The latter can be valuable for enhanced liner stability. With the MFL a net rotational motion to the liner can be readily produced from a small directional offset applied to each macron launcher. Such liner rotation has been demonstrated to provide stability to the Raleigh-Taylor modes that can limit the stable burn period at peak compression [4].

The elemental composition and shape of the macron will be considered later, but for this discussion its radial size and mass are the relevant parameters. The scale and number of macron launchers required for the liner can be readily estimated by what was investigated in the MTF liner experiments performed at AFRL [5]. Here a conventional Aluminum shell liner of roughly $300 \mathrm{~g}$ mass was imploded using the 4.5 MJ Shiva Star capacitor bank. The $5 \mathrm{~cm}$ radius liner was compressed with an axial current of $12 \mathrm{MA}$ at $84 \mathrm{kV}$ and produced a final liner kinetic energy of $\sim 1 \mathrm{MJ}$ in $22 \mu \mathrm{sec}$ [6]. Employing the macron launcher it should be possible to exceed this liner energy by a factor of three for a similar stored energy. For example, the target parameters for each launcher are a macron mass of $2 \mathrm{~g}$ accelerated to a velocity of $3 \mathrm{~km} / \mathrm{s}(3 \mathrm{~mm} / \mu \mathrm{s})$. The kinetic energy of each macron would thus be $\sim 10 \mathrm{~kJ}$. For a $1 \mathrm{~cm}$ radius macron, the 30 macron launchers depicted in Fig. 1 would converge to make contact at a radius of roughly $10 \mathrm{~cm}$ and be well merged at $5 \mathrm{~cm}$. Ten such rings arranged axially would form a liner of length comparable to the AFRL liner, and have roughly twice the mass $(0.6 \mathrm{~kg})$. The liner kinetic energy however would total $3 \mathrm{MJ}$. And it would compress from $5 \mathrm{~cm}$ radius to the minimum $(\mathrm{r}<2 \mathrm{~mm})$ in less time $(\sim 15 \mu \mathrm{sec})$. Without electrical contacts, the experimental apparatus and vacuum system can be greatly simplified. Difficulties with the post implosion vacuum integrity are also much easier to avoid.

It should be noted that the aperture in the chamber wall required for macron introduction is quite small $\left(\sim 3 \mathrm{~cm}^{2}\right)$, so that even with a few hundred such holes, the portion of reactor wall area exposed would be less than a small fraction of a percent of the total area. The fluence to the launcher structure is also significantly reduced by being completely outside the blanket with only a small acceptance angle for exposure due to the long connection tube length through the blanket.

It is not hard to see how the distributed launcher array greatly reduces the demand on the power delivery systems. With a constant acceleration of the macron to $3 \mathrm{~km} / \mathrm{s}$ over a distance of $1.5 \mathrm{~m}$, the liner energy input can occur over a time span as long as $1 \mathrm{msec}$ - two orders of magnitude slower than the conventional $\mathrm{Z}$ pinch method. As will be seen, the energy input from each macron launcher is low enough and slow enough that it can be provided by sub-kilovolt supplies with off-the-shelf solid state electronics. The driver circuits developed at MSNW that would be adapted for use on the macron launcher have demonstrated electrical efficiencies approaching $95 \%$. 
There are of course issues that must be settled for the macron formed liner concept to be validated. The principle questions, and the ones to be addressed in the work to be outlined here, are: (1) What is the timing window and acceptable velocity spread of the macron array that still delivers the desired liner behavior. (2) What is the dynamical behavior of the liner during compression, specifically how is it influenced by non-uniformities or rotation. (3) What is the optimal projectile geometry, trajectory and number. While the answers to these questions follow a basic sequential order, they are highly coupled. The most critical issue is to demonstrate the ability to produce the desired macron kinetic behavior in a consistent and repeatable manner. This will be the major goal of the phase I effort. Once this is achieved, a test of a complete azimuthal array could then be pursued. In parallel with this undertaking, a modeling effort will be initiated to guide in the design of macrons as well as the arrays. The testing of a full array would then be performed in phase II. Successful compression of an axial guide field would validate the concept, and full compression of the merged FRC would be the goal of a phase III effort. A key element in all this is the macron launcher itself. A discussion of what is thought at this point to be the most promising approach, and the one to be adopted for this effort will now be discussed.

\section{B. The Macron Launcher}

It is clear from the considerations above; the launcher must be simple, reliable and robust. It is believed that the prototype design to be tested possesses all these features. There is a wide array of possible methods to achieve the desired velocities and masses. The velocity requirement is rather modest compared to that attained by a large range of projectile acceleration methods. There are both electromagnetic (EM) and gas dynamic approaches that have achieved velocities far in excess of the $3 \mathrm{~km} / \mathrm{s}$ target velocity. Twostage light gas guns can accelerate $1 \mathrm{~g}$ aluminum spheres to velocities of $8 \mathrm{~km} / \mathrm{s}$ [7]. Rail guns have achieved similar velocities with much larger masses [8]. Pulsed inductive coils [9] and coil guns [10] are also possible devices. It turns out that however none of these devices can meet all the attributes desired for the launcher. For instance, the light gas gun requires large and fairly complicated firing arrangement, and typically employs chemical explosives as a first stage. It would thus be unlikely that an array of such devices that could achieve the timing jitter required for the liner assembly. As a crude estimate of what timing would be required, consider the arrival of a $1 \mathrm{~cm}$ long macron at the point of contact. If a position accuracy of $1 \mathrm{~mm}$ was desired, the firing time jitter at a macron closing speed of $3 \mathrm{~km} / \mathrm{s}$ would be $\sim 0.3 \mu \mathrm{s}$. While larger variations may prove to be acceptable, it will certainly be of this order. While this is an easily achieved jitter for EM launch techniques, it is probably too severe for gas dynamic
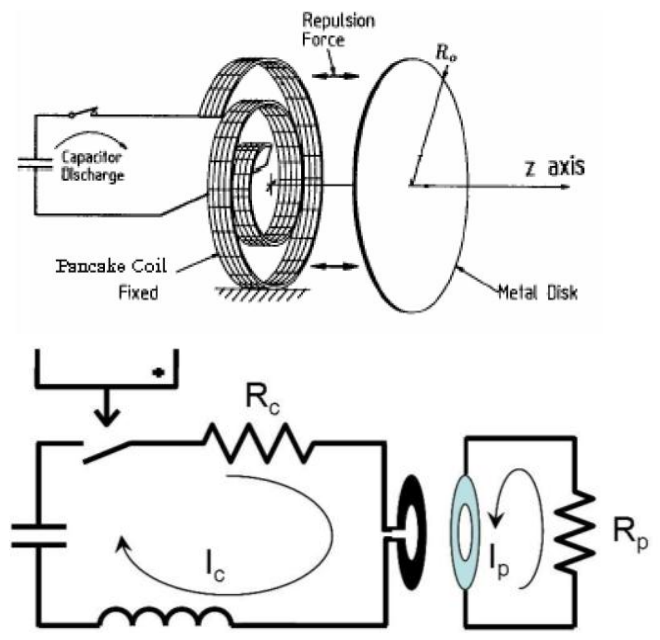

$$
\begin{gathered}
V_{c}=R_{c} I_{p}+\left(L_{d}+L_{c}\right) \frac{d I_{c}}{d t}+\frac{d\left(M_{c p} I_{p}\right)}{d t} \\
0=I_{p} R_{p}+\frac{d I_{p}}{d t}+\frac{d\left(M_{c p} I_{c}\right)}{d t}
\end{gathered}
$$

Figure 3. Pancake coil inductive launcher. Equation of motion (Eq. 1) together with the circuit equations above determine macron motion. 
approaches. Therefore only EM techniques will be considered. Achieving velocity control in addition eliminates techniques such as rail guns where rail contact and other issues limit the accuracy that can be achieved in terminal velocity. Rail guns have been pursued for many years for goals as diverse as directed energy weapons to space launch. There are two significant problems for the rail gun. It has poor energy coupling efficiency, and the arc nature of the current commutation makes long term repetitive pulsing very difficult. Purely inductive coupling eliminates most of the drawbacks of the rail gun. The coil gun employs a ferromagnetic projectile and can theoretically achieve velocities of up to $2 \mathrm{~km} / \mathrm{s}$. Core saturation and heating are the limiting factors. However the requirement that the macron be ferromagnetic is also very constrictive.

With the inductive accelerator, the motion of the macron is controlled by the propagating magnetic wave which can be made identical in each accelerator. Variation in macron response to acceleration due to variations in size or mass is largely self-correcting due to the axial gradient in the force imparted by the propagating field. If the macron moves out ahead of the wave the axial driving force experienced by the macron diminishes bringing the macron back into alignment. The same effect corrects for a macron that falls behind requiring greater force.

The force on the projectile in an inductive accelerator can be easily is derived from Lens' law with an equation of motion that depends on the coil-projectile geometry and currents:

$$
F(z, t)=I_{c}(t) \cdot I_{p}(t) \frac{d M_{c p}}{d z},
$$

where $\mathrm{M}_{\mathrm{cp}}$ is the mutual coupling between coil and projectile. It is actually possible to achieve very high velocities from a single pulsed inductive coil (see Fig. 3). Large velocities ( $\sim \mathrm{km} / \mathrm{s}$ ) were obtained from such an arrangement as depicted in Fig. 3 for a $2 \mathrm{~g}$ Aluminum washer. The problem with a single stage launch to high velocity is the required concentration of power in time and space makes repetitive pulsing very problematic. During launch the pulse coil undergoes tremendous forces where joule heating and fatigue would be major concerns. For more modest launch velocities $(\sim 500 \mathrm{~m} / \mathrm{s})$ repetitive operation is much more manageable, and has been achieved. What is clearly required is the staged inductive acceleration of the macron. In this manner the kinetic energy can be added incrementally avoiding the power concentration issues. The pulsed inductive acceleration technique (see Fig. 4) is very similar to that employed in the FRC acceleration and merging experiments at MSNW on the Inductive Plasma Accelerator (IPA) [2]. In this case the terminal FRC velocity exceeded $300 \mathrm{~km} / \mathrm{s}$. Here the two identical FRCs must arrive at the

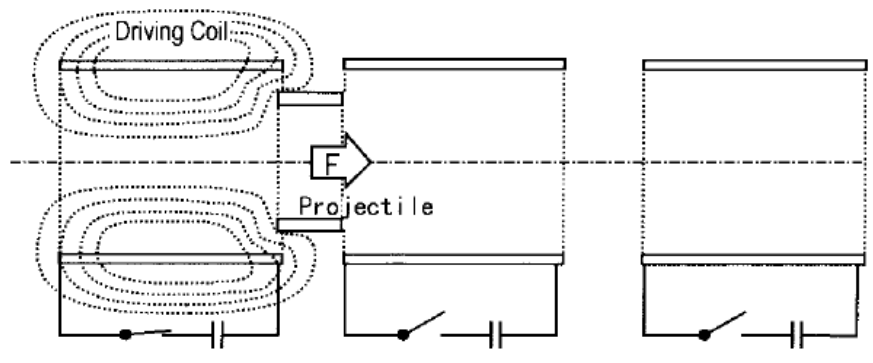

Figure 4. Pulsed inductive acceleration of a macron. experimental midplane within a fraction of a microsecond. Even this extreme case of merging has been achieved resulting in a stable, hot long-lived FRC. The technological challenge presented by macron merging should be far less demanding. 
Integrating the Eq. (1) along the axis of motion yields the work done on the projectile and thus the final kinetic energy of the projectile:

$$
\frac{1}{2} \mathrm{~m}_{\mathrm{p}} \mathrm{v}_{\mathrm{p}}^{2}=\mathrm{I}_{\mathrm{c}} \mathrm{I}_{\mathrm{p}} \int_{0}^{\infty} \mathrm{M}_{\mathrm{cp}} \mathrm{dz}=\frac{\mathrm{I}^{2}}{2} \mathrm{M}_{\mathrm{cp} 0}
$$

where $\mathrm{M}_{\mathrm{cp0}}$ is the value of the average mutual coupling to the array of coils experienced by the projectile along the length of the accelerator. It was assumed during the projectile acceleration that the current in the coil is oscillatory, and that the macron image currents completely screen the solenoidal coil fields $\left(I_{p}=I_{c} \equiv I\right)$. To maximize the efficiency of the driver, the drive coil circuits execute a full period before the circuit is opened. This allows any energy not transferred into macron motion to be returned to the capacitor for future use. The direction of the axial force is independent of field direction so that the axial force is continued with current reversal. By using an oscillatory current the magnetic field is kept from penetrating the macron and reducing the accelerating magnetic gradient. The polarity of each coil is reversed and timed so that the field is in alignment with the field produced during the previous coil's second half cycle. The stability of the macron is also much improved with this acceleration method as the direction of the dipole moment alternates as the macron moves from coil to coil preventing a possible wobble or tilt instability from growing. A multipole (quadrupole) guide field can also be applied to assure alignment of the macron and to maintain clearance with the accelerator wall.

\section{Magneto-Inertial Fusion Scaling}

While it is clear that a successful application of MFL could equal or exceed the compression that is attainable with a large scale, high voltage $\mathrm{Z}$ pinch, the question remains as to what is the best match of mass and energy to maximize the fusion gain. The MFL is unique in this way as these are both free parameters. Previous analysis has shown that it may be possible to accomplish breakeven at even sub-megajoule energies [11] Not surprisingly it requires operation at very small scale. Certainly MIF has significant advantages in terms of reactor power density and size, but technological challenges and low driver efficiencies can easily remove these advantages if care is not given to the manner which MIF is to be employed. To have a realistic hope of inexpensive and repetitive operation, it is essential to have the highest yield with the minimum liner kinetic energy. At small scale the implosion speed must be reasonably fast to maintain the magnetized plasma (e.g. FRC) equilibrium during compression. It would be hard to argue that it is not of paramount advantage to employ a closed field line plasma that has intrinsically high beta, and can be readily translated and compressed as the primary target plasma for MIF. Of all fusion reactor embodiments, only the FRC has the linear geometry high plasma $\beta$, and closed field confinement required for magnetic fusion at high energy density. Most importantly, the FRC has already demonstrated the confinement scaling with size and density required to assure sufficient lifetime to survive the compression timescale required for MIF over a wide range of conditions $[12,13]$. Thus the target plasma for the macron liner experiments to be employed here will be the FRC. It is worthwhile to give a short analysis of the Lawson criteria for MIF with an FRC target in order to determine the trade-offs between liner mass and energy.

For this analysis cylindrical symmetry will be assumed with the axial magnetic field as the primary confining field (a prolate FRC). For the FRC in this geometry the peak plasma pressure is equal to the external magnetic field pressure. It will also be assumed that the plasma density is 
adjusted so that at maximum compression the plasma temperature is $\sim 10 \mathrm{keV}$. From radial pressure balance one has:

$$
\mathrm{n}_{0}=\frac{\mathrm{B}_{0}^{2}}{2 \mu_{0} \mathrm{kT}}=2.5 \times 10^{20} \mathrm{~B}_{0}^{2},
$$

where the zero subscript indicates values at peak compression. It will be assumed that the liner is incompressible and that the liner radial implosion kinetic energy per unit length, $\mathrm{E}_{\mathrm{k}}$, is transferred into compression of both the FRC and axial magnetic field energy with no losses, i.e.:

$$
\mathrm{E}_{\mathrm{k}}=\frac{1}{2} \mathrm{M}_{\mathrm{L}} \mathrm{v}_{\mathrm{m}}^{2}=\frac{\mathrm{B}_{0}^{2}}{2 \mu_{0}} \pi \mathrm{r}_{0}^{2} .
$$

where $M_{L}$ is the liner mass per unit length. The liner dwell time, $\tau_{D}$, is characterized by the terminal liner velocity, $\mathrm{v}_{\mathrm{m}}$, and the minimum liner radius, $\mathrm{r}_{0}$,

$$
\tau_{\mathrm{D}} \sim \frac{2 \mathrm{r}_{0}}{\mathrm{v}_{\mathrm{m}}}
$$

Using Eq. (4) to solve for $\mathrm{v}_{\mathrm{m}}$, one has for the Lawson criteria for the FRC based MIF:

$$
\mathrm{n} \tau \sim \mathrm{n}_{0} \tau_{\mathrm{D}}=3.2 \times 10^{17} \mathrm{~B}_{0} \sqrt{\mathrm{M}_{\mathrm{L}}} .
$$

There are several notable conclusions one can draw from this expression. First, there is no explicit dependence on the liner material, its density or conductivity. More significantly, there is no explicit size dependence. There can thus be a very wide range of liner masses and materials that could be employed to achieve fusion gain. Equation (6) can be restated in terms of liner kinetic energy per unit length:

$$
\mathrm{n} \tau=2.8 \times 10^{14} \frac{\sqrt{\mathrm{E}_{\mathrm{K}} \mathrm{M}_{\mathrm{L}}}}{\mathrm{r}_{0}} .
$$

There are limits of course to the magnitude that one can achieve for all these parameters. Clearly the smaller the scale the better as this minimizes the total energy delivered to the blanket and structural elements at high Q. A liner radial convergence similar to the AFRL results would yield a final radius of $\mathrm{r}_{0} \sim 2 \mathrm{~mm}$. Recall the target macron liner energy $(3 \mathrm{MJ})$ and mass $(0.6 \mathrm{~kg})$. Using macron trajectories that provide enough axial compression $(3 \mathrm{x})$ to match the FRC equilibrium contraction results in a final liner length of $5 \mathrm{~cm}$. With these values the resultant $\mathrm{n} \tau$ product from Eq. (7) is $\sim 4 \times 10^{21} \mathrm{~m}^{-3}$-s which provides for sufficient margin to make the MFL a significant fusion energy source.

\section{Macron liner composition}

Small scale does carry with it certain implications. The liner implosion speed must be reasonably fast to maintain the FRC equilibrium during compression. Vaporization of the liner during implosion significantly increases the liner resistance and dramatically reduces its ability to trap and compress the magnetic field. It was first pointed out by Cnare in his landmark foil compression experiments [14] that this process can limit the ultimate macron liner velocity (energy) that can be attained. The material properties relating to this heating (electrical conductivity, melting point, heat capacity, etc.) can be characterized by a parameter $\mathrm{g}_{\text {mat }}$ defined by the "current integral": 


$$
\int_{0}^{t_{m}} I^{2} d t=g_{m a t} A^{2}
$$

where $\mathrm{I}$ is the liner current flowing through the material cross-sectional area, A, in the direction of current flow. Normalizing to the action constant for the vaporization of aluminum from 300 ${ }^{\circ} \mathrm{K}$ one has for the maximum velocity:

$$
\mathrm{v}_{\mathrm{m}}=6.8 \times 10^{10} \mathrm{~g}_{\mathrm{Al}} \frac{\delta_{\mathrm{L}}}{\rho_{\mathrm{L}}},
$$

where $\rho_{\mathrm{L}}$ is the macron material density and $\delta_{\mathrm{L}}$ is the liner thickness. While this is not a significant limit during compression due to the expected liner thickness, it can limit the velocity that can be imparted to the macron. The maximum velocity for various materials is given in Fig. 5.

There are several promising materials for use as a macron. A lithium macron would be

\begin{tabular}{|lcc|cc|}
\hline \multirow{2}{*}{ Element } & Current Intgrl. & Ratio to $\mathbf{A l}$ & $\mathbf{V}_{\mathbf{m a x}} / \boldsymbol{\delta}(\mathbf{m} / \mathbf{s})$ & Ratio to Al \\
\cline { 4 - 5 } $\mathrm{Ag}$ & $1.53 \mathrm{E}+17$ & 1.51 & $9.19 \mathrm{E}+06$ & 0.39 \\
$\mathrm{Al}$ & $1.01 \mathrm{E}+17$ & 1.00 & $2.37 \mathrm{E}+07$ & 1.00 \\
$\mathrm{Al}$ & & & & \\
$(6061)$ & $1.07 \mathrm{E}+17$ & 1.05 & $2.49 \mathrm{E}+07$ & 1.05 \\
$\mathrm{Au}$ & $1.39 \mathrm{E}+17$ & 1.37 & $4.53 \mathrm{E}+06$ & 0.19 \\
$\mathrm{Be}$ & $9.24 \mathrm{E}+16$ & 0.91 & $3.15 \mathrm{E}+07$ & 1.33 \\
$\mathrm{Cu}$ & $\mathbf{2 . 3 3 \mathrm { E } + 1 7}$ & $\mathbf{2 . 3 0}$ & $1.64 \mathrm{E}+07$ & 0.69 \\
$\mathrm{Li}$ & $1.36 \mathrm{E}+16$ & 0.13 & $1.61 \mathrm{E}+07$ & 0.68 \\
$\mathrm{Na}$ & $1.19 \mathrm{E}+16$ & 0.12 & $7.70 \mathrm{E}+06$ & 0.33 \\
$\mathrm{~Pb}$ & $5.79 \mathrm{E}+15$ & 0.06 & $3.21 \mathrm{E}+05$ & 0.01 \\
$\mathrm{Mg}$ & $2.20 \mathrm{E}+16$ & 0.22 & $7.95 \mathrm{E}+06$ & 0.34 \\
\hline
\end{tabular}

Figure 5. Candidate macron liner materials blanket material. After fusion burn and disassembly it could then be reabsorbed into the blanket with each pulse.

\section{E. Target FRC physics}

From Fig. 5 it is clear that the nominal macron liner target velocity of $3 \mathrm{~km} / \mathrm{s}$ is well below the velocity limitation from melting for a material thickness of $1 \mathrm{~mm}$ or more for almost any material of interest. It is important to know whether this compression speed is consistent with the FRC formation, translation and equilibrium lifetimes. FRC lifetime scaling obtained from previous experiments stated in terms of externally measured parameters was found to be $[12,15]$ :

$$
\tau_{\mathrm{N}}=3.2 \times 10^{-15} \varepsilon^{0.5} \mathrm{x}_{\mathrm{s}}^{0.8} \mathrm{r}_{\mathrm{s}}^{2.1} \mathrm{n}^{0.6}
$$

Where $\varepsilon$ is the FRC elongation, $\mathrm{x}_{\mathrm{s}}$ the ratio of the $\mathrm{FRC}$ radius, $\mathrm{r}_{\mathrm{s}}$, and the coil radius, $\mathrm{r}_{\mathrm{c}}$. As is often done, a rigid rotor current profile is assumed, so that one can restate this scaling in terms of only the FRC poloidal flux and the external magnetic field:

$$
\tau_{\mathrm{N}}=0.03 \varphi_{\mathrm{p}}^{0.9} \mathrm{~B}^{-0.2} .
$$


Typical values for FRCs formed in a $10 \mathrm{~cm}$ coil are $\varphi_{\mathrm{p}} \sim 1.5 \mathrm{mWb}$ at $0.7 \mathrm{~T}$ for a particle lifetime of $90 \mu \mathrm{sec}$. One can expect FRC lifetime to decrease as the FRC is compressed. From Eq. (11) the lifetime would decrease to $34 \mu \mathrm{sec}$ at a megagauss field $\left(\mathrm{B}_{0}=100 \mathrm{~T}\right)$. A safe value for the compression time would thus be $<25 \mu \mathrm{sec}$. With the macron formed liner method the maximum radial velocity is attained at the exit of the launcher. While the FRC traversal of the burn chamber is much less than the FRC lifetime, the macron traversal of the burn chamber will no doubt be significantly longer than the FRC lifetime. Therefore the injection of the FRC

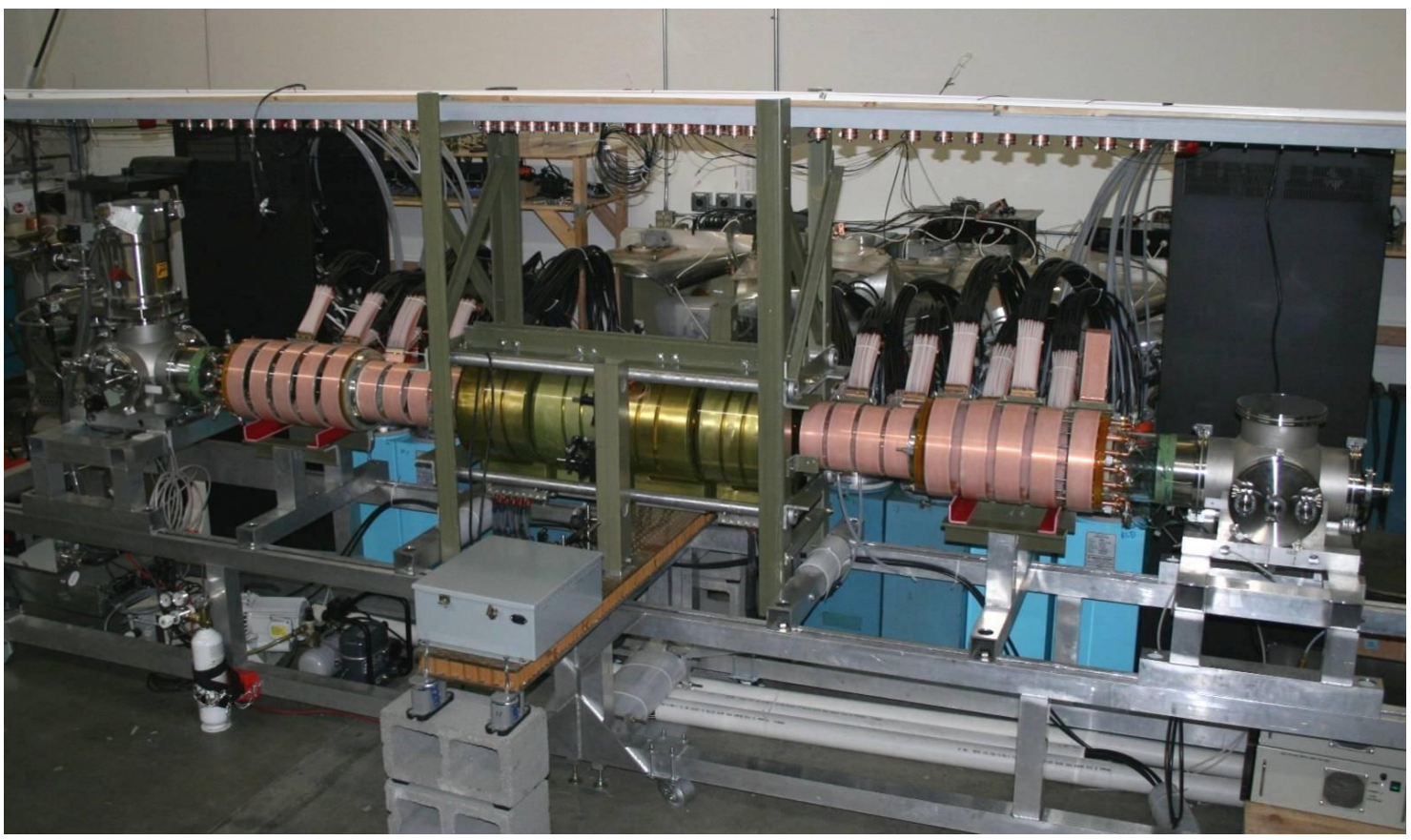

Figure 6. IPA-C experiment with new source, accelerator and compression system upgrades.

would be held off until the liner has been formed and developed some flux compression as depicted in Fig. 1.

As mentioned, FRC formation, acceleration and merging of FRCs of appropriate size and energy density for the MFL has been already been developed at MSNW on the Inductive Plasma Accelerator (IPA) device. The device consists of two inductive plasma accelerators. In these experiments a high density FRC plasmoid was formed and accelerated out of each accelerator into a merging/compression chamber resulting in a stable, stationary, high temperature ( $\mathrm{T}$ $500 \mathrm{eV})$ FRC plasmoid. In the IPA the acceleration of the FRC plasmoid is through the electromagnetic interaction of the radial magnetic field of the sequentially activated accelerator coils (see Fig. 6), and the large, induced FRC toroidal plasma current (i.e. the Lorentz force). After the initial results on IPA a larger FRC source and magnetic compression bank was added to increase the FRC energy density. The merged FRC was then simultaneously compressed achieving a temperature over $1.5 \mathrm{keV}$ and exhibited lifetimes better than past FRC scaling with an $\mathrm{n} \tau \mathrm{T}_{\mathrm{i}}=1.2 \times 10^{17} \mathrm{~m}^{-3}-\mathrm{keV}$-s. This result was obtained in a FRC plasma with a separatrix radius of just $3 \mathrm{~cm}$ employing a fairly modest compression field (B $\sim 1 \mathrm{~T}$ limited by the available bank energy). The current effort is aimed at increasing this compression several fold, but the key 
finding for the MFL approach was that FRCs can be accelerated at a rate of $4.6 \times 10^{10} \mathrm{~m} / \mathrm{s}^{2}$, merged and compressed without loss of stability or confinement.

The macron liner compression heating of the FRC plasma has the potential to be much more efficient than other methods. The electrical efficiency of both the FRC accelerator and macron launcher can be quite high $(>90 \%)$ if active switching is employed. For the launcher it will be shown that such active switching can be readily accomplished due to the low coil voltages required for macron acceleration. The challenge for the FRC accelerator is energy recovery at the higher voltages $(\sim 25 \mathrm{kV})$ employed in the plasma accelerator. There are several new switch technologies (thyratrons and HV diodes) that make even the plasma component capable of recuperative energy recovery. Since the bulk of the energy input is in the form of the liner energy, it is not as critical that the plasma accelerator be highly efficient, but achieving this does make repetitive operation that much easier.

\section{Anticipated Public Benefits}

(1) Establish a new approach to fusion energy that avoids the costly development of current low density systems.

The advancement of small scale pulsed fusion embodied in the macron formed liner compression concept would provide for a near term, low cost, and practical path for achieving high gain fusion conditions. If all the planned phases prove successful it would establish the concept well beyond the "proof of principle" level and would justify further development into a fusion gain device. In addition to the obvious benefit of directly providing for a new path to a commercially viable source of fusion energy, the work would be of direct and possible critical benefit to other fusion pursuits now supported by the Department of Energy. Specifically:

(2) Provide a powerful method for plasma compression to facilitate scientific understanding of high density plasma states.

There are several DOE programs investigating the FRC for fusion over a large range of plasma densities and conditions. At the highest densities the Magnetized Target Fusion (MTF) experiments at Los Alamos National Laboratory (LANL) [16] and the Air Force Research Laboratory (AFRL) [6] represent an attempt to compress plasmas to fusion conditions using a conducting metal liner. The experiment to be performed is a very ambitious one that would amount to a major advance if successful. The macron formed liner compression approach, combined with the formation, translation and merging of FRCs already developed at MSNW, would provide a low cost, highly efficient method for producing even higher energy density states. Without the need for a close fitting liner and power delivery system, the destructive aspects from imploding a metallic liner as massive as that planned for the MTF experiment at AFRL could be avoided. This would allow for greater diagnostic access, and more extensive testing.

(3) Enable a wide range of fusion energy applications not easily accessible to current fusion approaches. 
The successful development of the macron formed liner compression of the FRC would provide for a practical approach to achieving a small scale, high yield source of fusion neutrons. At a minimum, this method will facilitate the exploration and development of a new regime of fusion plasma physics that could lead to very different application and usage to that of the path now being pursued by virtually all other fusion efforts. The neutron diagnostic applications would be numerous as it has been for the neutron spallation sources, but at a fraction of the cost and size. Even with development of only a Q 1 MFL system there would be application to the breeding of fissile fuel, particularly for the Thorium cycle, to support the future generation of advanced fission plants. There would also be the application of burning and transmuting of longlived fission products and actinides accumulated from past commercial fission. 


\section{Degree to which Phase I has Demonstrated Technical Feasibility}

\section{A. Numerical Modeling Results}

The 3-D colliding macron liner was simulated using ANSYS Multiphysics which has the capability of calculating the behavior of the colliding aluminum macrons well into the non-linear
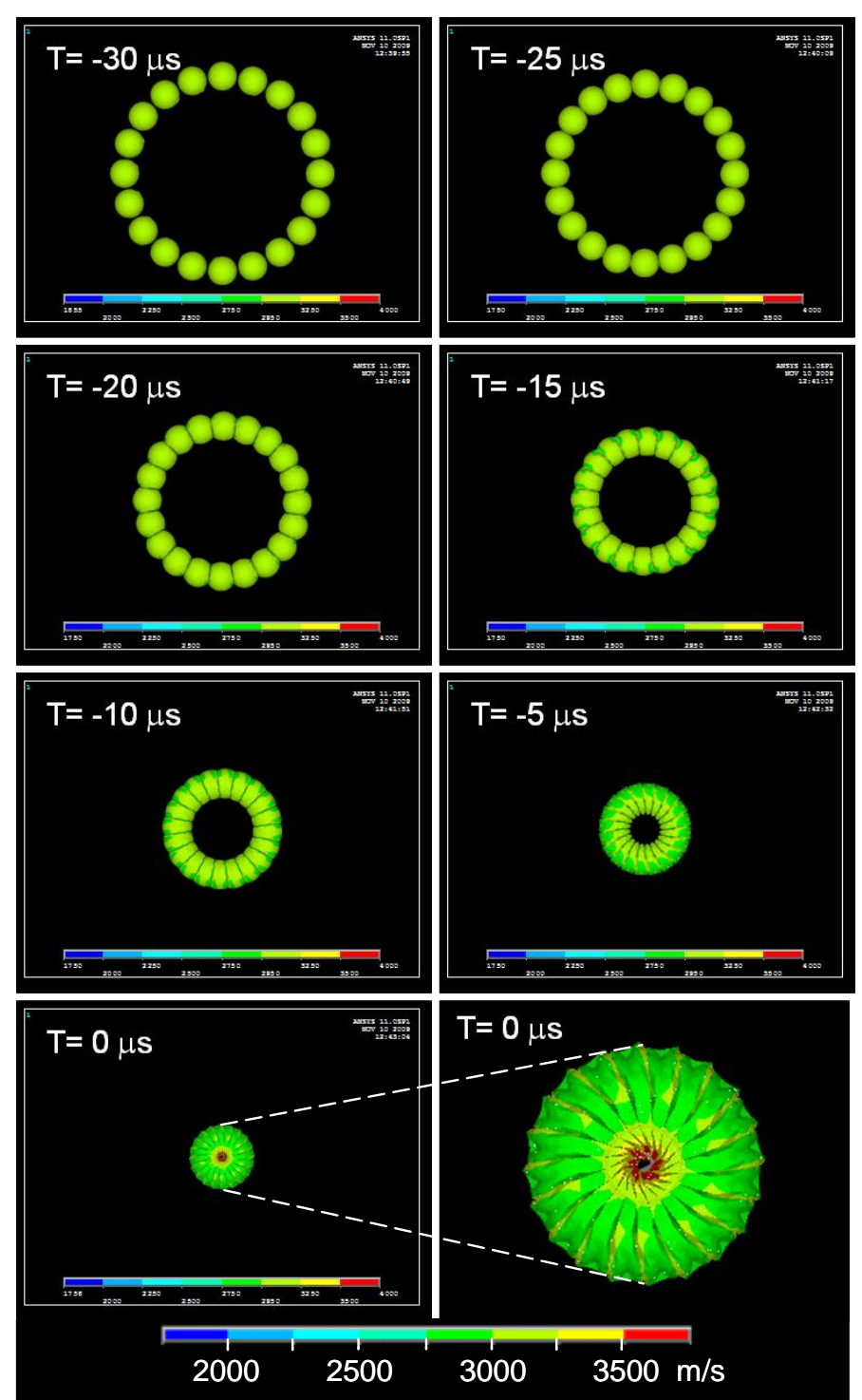

Figure 7. Macron velocity profiles during formation and compression of a macron ring with radial directional energy of $0.6 \mathrm{MJ}$. The liner segment is formed by initiating 20 hollow spherical aluminum macrons moving radially inward at 3 $\mathrm{km} / \mathrm{sec}$ with the center of mass $5 \mathrm{~mm}$ off-axis to impart angular momentum to the liner for stabilization. plastic deformation regime. The initial calculations were performed for macrons comprised of hollow aluminum spheres, 6.7 grams in mass which were launched radially inward at $3 \mathrm{~km} / \mathrm{sec}$. The spheres were observed to collide, deform, and finally form a complete, reasonably uniform liner at small radius. The entire process occurred with little diminution in the radial velocity as the thin walled, hollow spheres expend little energy in yielding under compression. For the target velocity of $3 \mathrm{~km} / \mathrm{sec}$ the macron merging and compression is sub-sonic. The simulation was 3 dimensional and both stabilizing rotation (initiated with small non-radial velocity component) and 3-D compression (using 2-3 arrays of macron liners) were also modeled. Finally, a complete treatment of the transient structural physics of the macron collision and integration was performed, including full plastic deformation stress-strain relationships, detailed surface roughness, and interaction studies.

Studies of liner uniformity and stability showed that the readily compressive nature of the hollow macrons themselves allows for a fairly large tolerance in initial velocity and timing, and still yield a uniform liner compression. It was found that initial positioning of up to one half macron radii ( $25 \%$ of total scale) is tolerable. For a $3 \mathrm{~m}$ diameter chamber this corresponds to an initial velocity and

timing accuracy of $15 \mathrm{~m} / \mathrm{s}$ and $2.5 \mu \mathrm{s}$. Surprisingly, it was found that even a fully missing macron is compensated for during the compression process as can be seen in Fig. 8. It was 

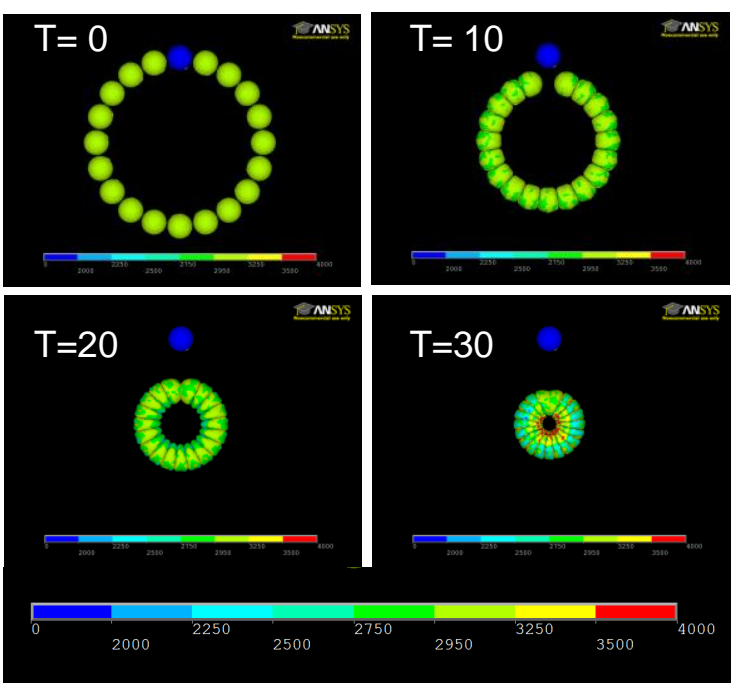

Figure 8. Macron velocity profiles with one macron initialized at zero velocity.

observed that greater than $95 \%$ of initial kinetic energy is maintained during maximum compression. A key advantage to this method of liner formation is the ability to aim the macrons to converge axially matching the FRC contraction and increasing the compressional energy on target. The macrons can also be aimed slightly off the axis of symmetry in order to provide rotational stability to the liner. The calculation shown in Fig. 7 illustrates the liner formation process employing 20 hollow aluminum macrons. In addition to the timing and velocity perturbations, the modeling showed that the high compressibility of the macrons allowed for significant rotation and axial variations to be added to the liner and still maintain maximum radial velocity. Phase II work will incorporate an axial magnetic field to determine the compressive efficiency of the MFL.

\section{B. Experimental Results}

Two experimental efforts were carried out during phase I. The original plan called for development and testing of a single multi-stage macron launcher using existing solid state switches and capacitors at MSNW. This task was accomplished and will be discussed shortly. The other endeavor was an attempt to gain insight into the macron merging process itself. Even though this was not part of the original plan, it was realized that the test stand constructed for the Foil Liner Compression (FLC) experiments at MSNW might allow for the possibility of imploding a ring of 'mini'-macrons using the theta pinch coil as the driver. The test setup for the foil liner is shown in Fig. 9. This device was constructed in order to test both the coil and foil behavior before implementation on a high vacuum system where the FRC formation chambers are made of
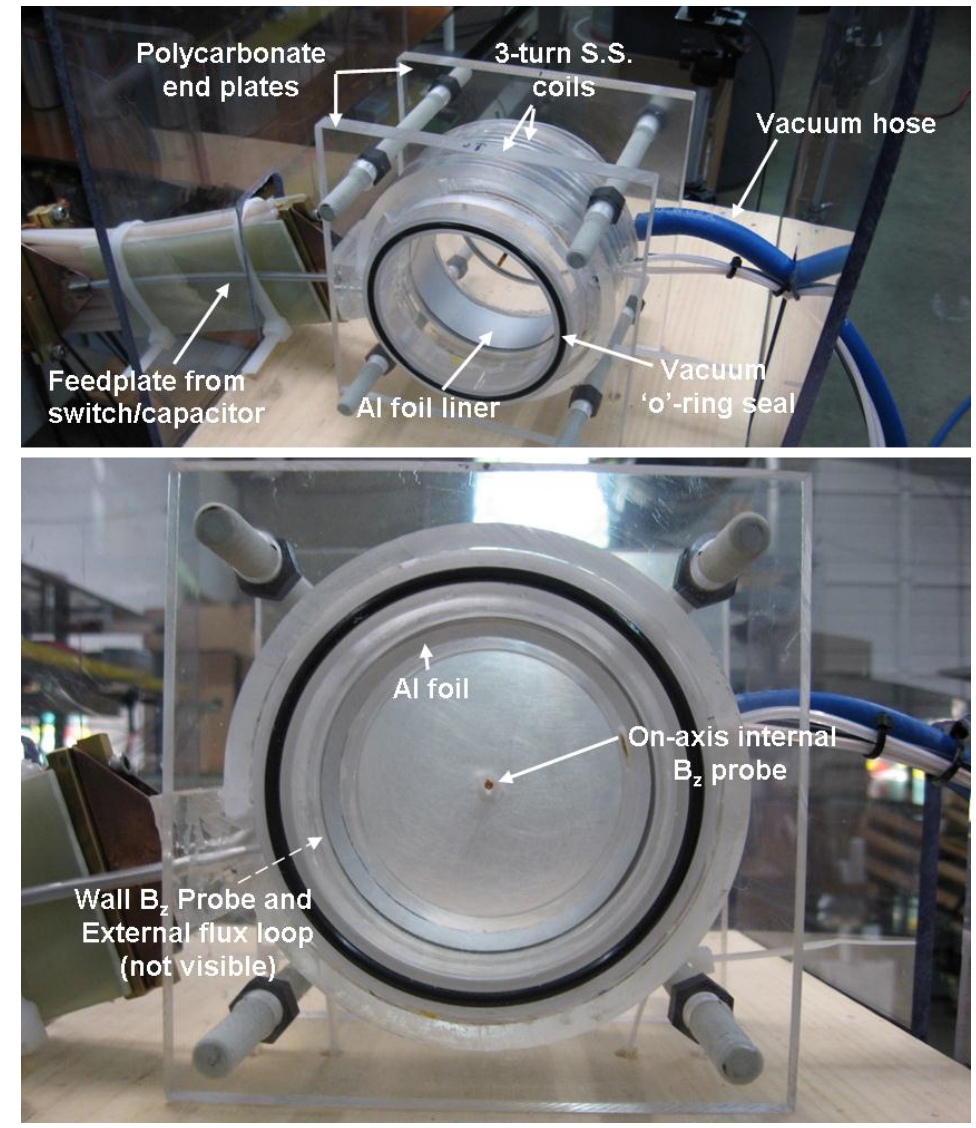

Figure 9. FLC liner test setup. (top) various elements of the setup indicate. (bottom) on axis view similar to fast framing camera. (Location of B probes indicated.) 
fused silica. The driver coil was split into left and right handed three-turn coils driven in parallel to improve coupling to the pair of $30 \mathrm{kV}, 17 \mu \mathrm{F}$ capacitors. In the initial testing at half power, 3 gram foils were accelerated to $1.3 \mathrm{~km} / \mathrm{sec}$ with a coupling efficiency of $23 \%$. The final coil will be larger for better coupling, and operated at higher power to achieve the target of $2 \mathrm{~km} / \mathrm{sec}$.

As was found with the staged acceleration of the macrons, the initial movement from rest is generally done with poorer coupling efficiency. The coupling to the macron becomes more

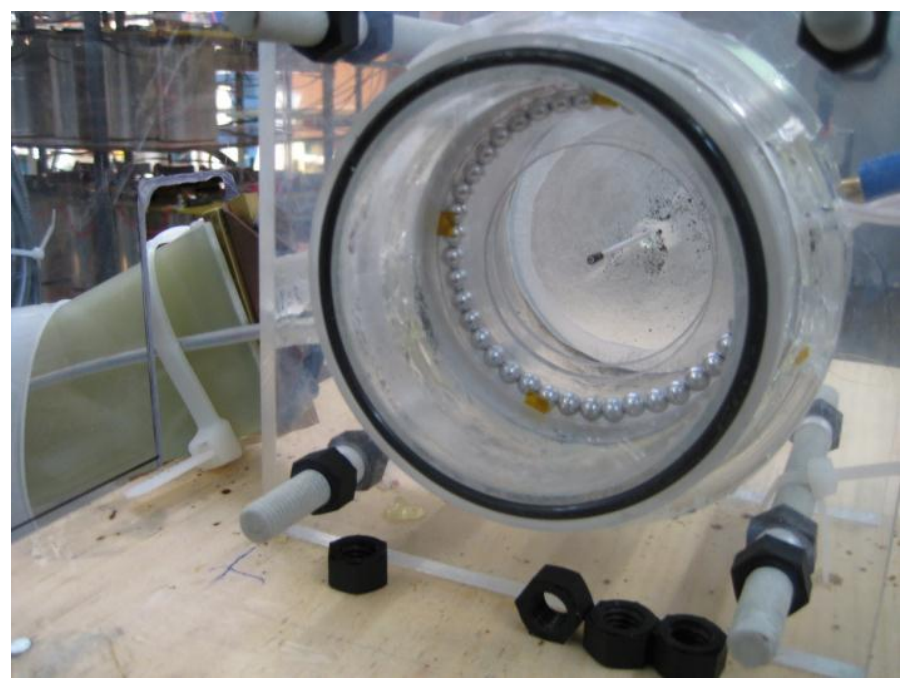

Figure 10. FLC test setup with $1 / 4$ " mini-macrons. efficient at higher macron velocities as the match to the magnetic waveform is better, and fundamentally the energy added scales with the macron velocity $(\mathrm{E} \sim \mathrm{v} \cdot \Delta \mathrm{v})$.

After successful testing with aluminum foils, the device became available for mini-macron ring experiments. It was possible to purchase small Aluminum 1/4" $(6.3 \mathrm{~mm})$ diameter Aluminum spheres for this test. It required 42 mini-macron spheres to complete a full ring at the inner chamber diameter of $8.4 \mathrm{~cm}$. A picture of the setup is shown in Fig. 10. A plastic 'race' and Kapton tape were used to hold the macrons in place against gravity. Given the precarious nature of the macron contacts, it was not possible to assure accurate alignment. A fine Aluminum wire was also used to help align the macrons. Despite some misalignments, the collapse and distortion for each macron after compression was virtually identical. More will be said on this point later.

It was clear from the beginning that the split three-turn coils designed to drive a cylindrical liner were far from an

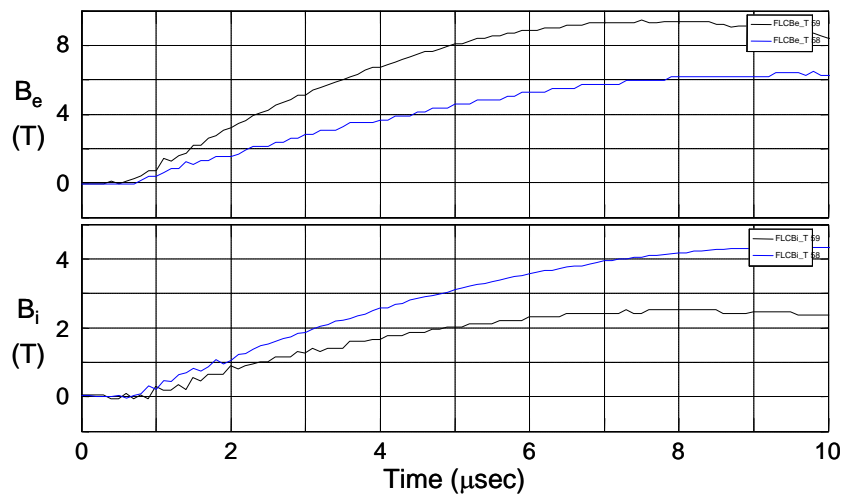

Figure 11. Time histories of the external magnetic field, $B_{e}$, and internal magnetic field, $\mathbf{B}_{\mathbf{i}}$. Blue traces are without cylindrical Aluminum ring (vacuum), and black traces are with the ring present.

ideal driver for the much narrower macron ring. In fact there was a gap in the driver coil precisely where one would have wanted the primary turn. The end turns were particularly ineffective in coupling to the macron ring. Flux leakage from axial diffusion of the magnetic field into the ring would also be much more rapid than for a cylindrical liner where the flux penetration is characterized primarily by the $\mathrm{L} / \mathrm{R}$ penetration time of the liner. In order to estimate the effect of this diffusion, a test was conducted with a short, thick Aluminum 
cylindrical ring $3 / 16 "(4.8 \mathrm{~mm})$ in axial extent. The results of the test are shown in Fig. 11. The ring was constructed thick enough to resist compression so that the changes in flux are the result of flux penetration only. As can be seen the field inside the ring is diminished by the presence of the ring (lower traces in Fig. 11), but a substantial field penetrates the ring. The screening effect of image currents in the ring is clearly observed by the significant increase in the field external to the ring (see upper traces in Fig. 11).

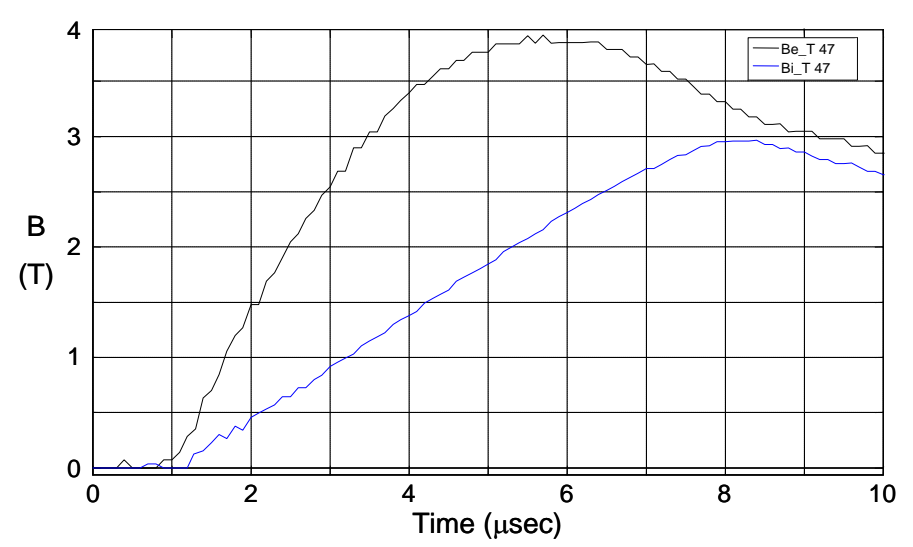

Figure 12. Time histories of the external magnetic field, $B_{\mathrm{e}}$ (blue trace), and internal magnetic field, $B_{i}$ (black trace) with fixed 42 element macron ring.

The same test was then performed with the mini-macron ring. In this case the ring was supported by a polycarbonate cylinder on the inside preventing collapse of the ring. The results are shown in Fig. 12. It can be seen that the macrons behave similar fashion. The compression bank was operated in a somewhat different manner to avoid undue forces on the macrons. The capacitance was reduced by half and the coil was also operated at lower voltage. This was apparently not sufficient as the macrons were not constrained axially and any small perturbation allowed the macrons to move axially. In fact a few of the macrons were observed to have been dislodged during the discharge. The rapid loss of field exclusion after $\sim 5 \mu \mathrm{sec}$ is likely due to the macrons losing contact and no longer forming a contiguous ring. In the actual implosion experiment the macron contact is strengthened as the macrons move in radially, which was prevented in this case by the polycarbonate inner cylinder.

The mini-macrons were inserted into the test chamber as pictured in Fig. 10. The macron formed ring radius can be determined from the three diagnostic loops that measure the internal field on axis $B_{i}$, the external field at the wall $\mathrm{B}_{\mathrm{e}}$, and the total flux inside the driver coil, F. These three quantities are related by the

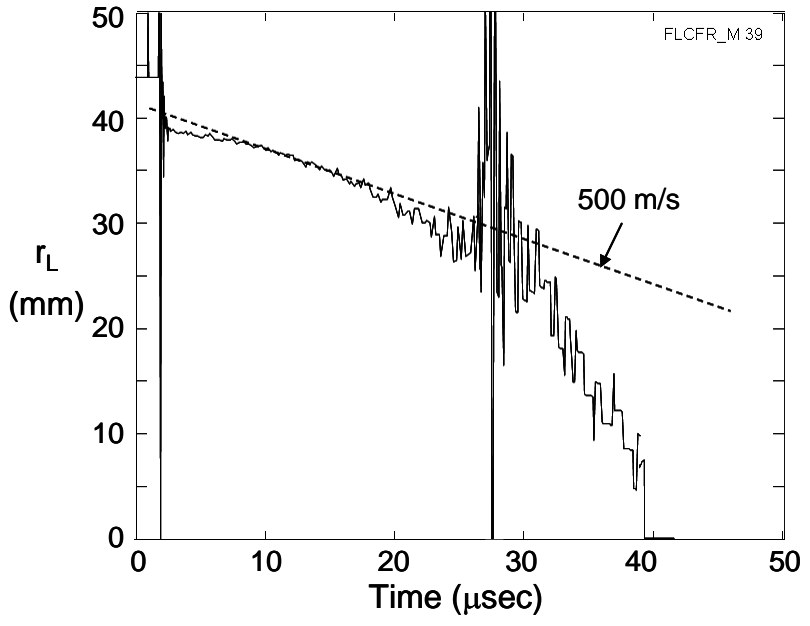

Figure 13. Macron ring radius inferred from magnetic field measurements and Eq. (xx). following equation:

$$
\mathrm{F}(\mathrm{t})=\mathrm{B}_{\mathrm{e}}(\mathrm{t}) \pi \mathrm{r}_{\text {loop }}^{2}+\mathrm{B}_{\mathrm{i}}(\mathrm{t}) \pi \mathrm{r}_{\mathrm{L}}^{2}(\mathrm{t})
$$


Knowing $\mathrm{r}_{\text {loop }}$ and given measurements of $\mathrm{B}_{\mathrm{e}}, \mathrm{B}_{\mathrm{i}}$, and $\mathrm{F}$, this equation can be solved for the only remaining unknown, the radius of the liner $r_{L}$. This equation assumes that there is no dissipation in the liner on the timescale of the compression. A reference shot with the cylindrical aluminum ring was used to help correct for finite geometry and flux leakage. The ring radius result for the mini-macron ring is found in Fig. 13. While the flux leakage is minor early on, as the ring slowed, flux leakage will make the inferred radius appear to shrink rapidly to zero. It is clear from Fig. 13 that after peak velocity was achieved $(\sim 15 \mu \mathrm{sec})$, the apparent rapid decrease in radial position is much more likely to be flux leakage than further acceleration of the macron ring as the external magnetic field has dropped precipitously by this time. A very electrically noisy event occurs at $\sim 27 \mu \mathrm{sec}$. This may be the signature of an electrical opening of the macron ring as the inferred radius plummets after this time.

The relatively low ring velocity inferred from the measurements was not surprising given the poor coil coupling geometry. Even with the rather low velocity observed, there were two main reasons that prevented the macrons from complete collapse as in Fig. 7. By using so many tiny spheres the contact area and number of contacts made for a loop resistance much higher than would be encountered in the prototype macron liner

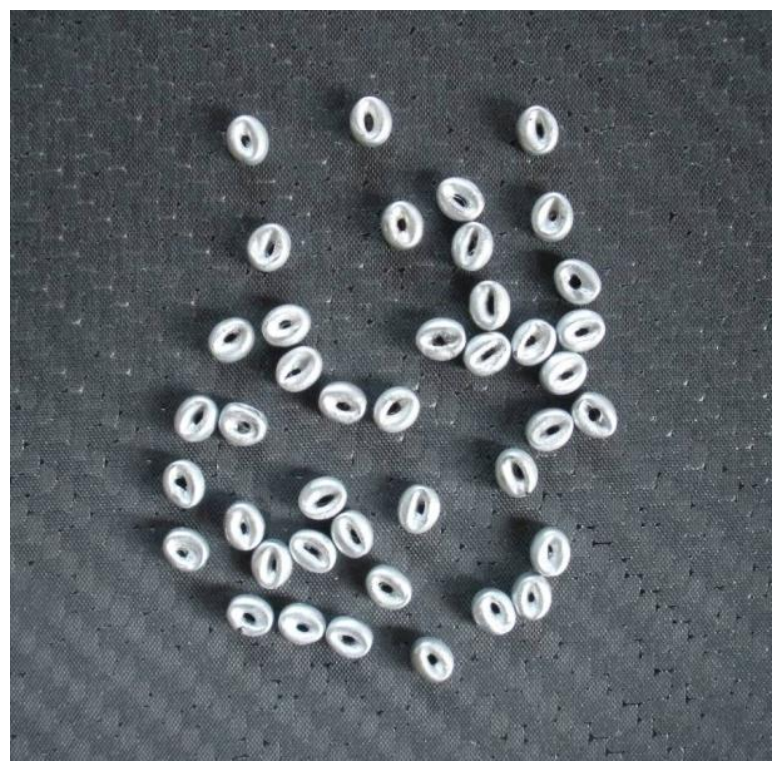

Figure 14. 1/4" diam. mini-macrons after compression. which will have roughly 20 times the contact surface area and as few as 20 macrons. The principal reason however is based on the same explanation as to why ants are proportionately much stronger than elephants. These little balls were very stiff to compression. The small size was dictated by the desire to keep the mass of the macrons roughly the same as the foils. The 42 macron ring weighed just 3.5 grams. The much larger, proportionately thinner-walled macrons to be employed in the prototype liner, and assumed in the numerical calculations, yield readily under compression with essentially no significant dissipation as illustrated in Fig. 7. A similar calculation using the dimensions and wall thickness of the smaller spheres showed that the kinetic energy was rapidly consumed in overcoming the buckling resistance of the tiny spheres.

From analysis of the macrons after compression it was observed that the macrons were compressed azimuthally by about a factor of two during the compression. A picture of the macrons gathered up after the implosion is shown in Fig. 14. What was remarkable was the uniformity of the compression. All of the macrons collapsed in the same way and to the same extent. A small 500 micron hole was drilled through each macron to evacuate the sphere as they were observed earlier to rupture when the air inside was heated due to the Ohmic heating of the aluminum. The balls were aligned by a fine wire threaded through the holes. After the discharge the holes appear greatly enlarged due to the inward buckling of the sphere at that point. The total mass of the macron ring was not measurably different after compression so that melting and vaporization of the macrons did not appear to be significant. As the sphere buckles, the contact 
area rapidly increased as the macrons merged, and the shiny edges indicate that these areas carried the azimuthal image currents as expected.

As mentioned, a numerical calculation based on the actual macron ring dimensions, macron number, mass, and size was carried out. It was initialized with the peak velocity $(500 \mathrm{~m} / \mathrm{s})$ inferred by the magnetic measurements. The results were essentially the same as the experimental results with the deceleration of the macrons as kinetic energy was converted into internal deformation work. The same distortions in the macrons seen in Fig. 14 were also observed for the macrons in the calculations. This is encouraging as it indicates the accuracy of the numerical calculations in predicting the actual results when given the corresponding initial conditions. 


\section{Macron Launcher Experiments}
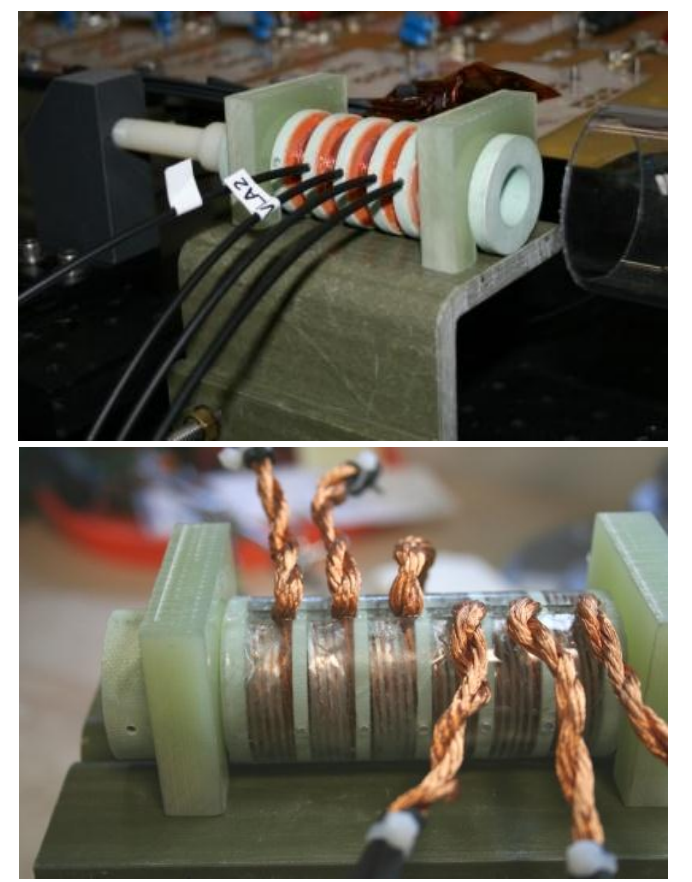

Figure 15. Six Stage, High-Q Macron Launcher. (Lower) Six epoxy-encapsulated, five-turn Litz wire coils wound on a hollow G-10 cylindrical form. (Upper) Highintensity $660 \mathrm{~nm}$ optical fiber optic for velocity measurements (200 ns resolution).

observed to be near the resolution of the digitizer at a fraction of a microsecond. The macron arrival times varied by less than $1 \mu \mathrm{sec}$ corresponding to a velocity accuracy of $2 \mathrm{~m} / \mathrm{s}$. Additionally, the velocity could be pre-programmed to accuracy of less than $5 \mathrm{~m} / \mathrm{s}$. An initial position study was performed as it was found that maintaining the initial position of the macron was important in minimizing the jitter as well as the final macron velocity. This is primarily due to the accelerator coil inefficiency at low macron velocities where the magnetic field oscillations were not
A six-stage macron launcher was constructed in order to determine the efficiency, timing precision, and positional accuracy of gram-scale pulsedinductive launchers. The initial launcher was constructed with six separate coil stages. Each $1.3 \mathrm{~cm}$ long stage consisted of a 5-turn $1.9 \mathrm{~cm}$ diameter coil made from 13 gauge Litz wire each potted in a lowviscosity epoxy. Each coil was driven with a $600 \mathrm{uF}$ electrolytic capacitor bank switched with six paralleled $1700 \mathrm{~V}$ Isolated Gate Bipolar Transistor (IGBT) switches producing a peak current of $24 \mathrm{kA}$ at $1 \mathrm{kV}$ resulting in an axial magnetic field of up to 25 Tesla with a cylindrical macron. Between the coils a high-intensity, $660 \mathrm{~nm}$ fiber transmitter/receiver was positioned for velocimetry measurements (see Fig 15). The initial launcher is shown in Fig. 15.

The six stage launcher successfully demonstrated the pulsed-inductive acceleration of cylindrical macrons with masses ranging from 1 to 4 grams. Variable aspect ratio cylinders and spheres were also examined. The six stage launcher demonstrated a peak velocity of $280 \mathrm{~m} / \mathrm{s}$ and validated performance expectations for the limited energies tested. Several important characteristics were exhibited. The jitter from sequential launches with different macrons was

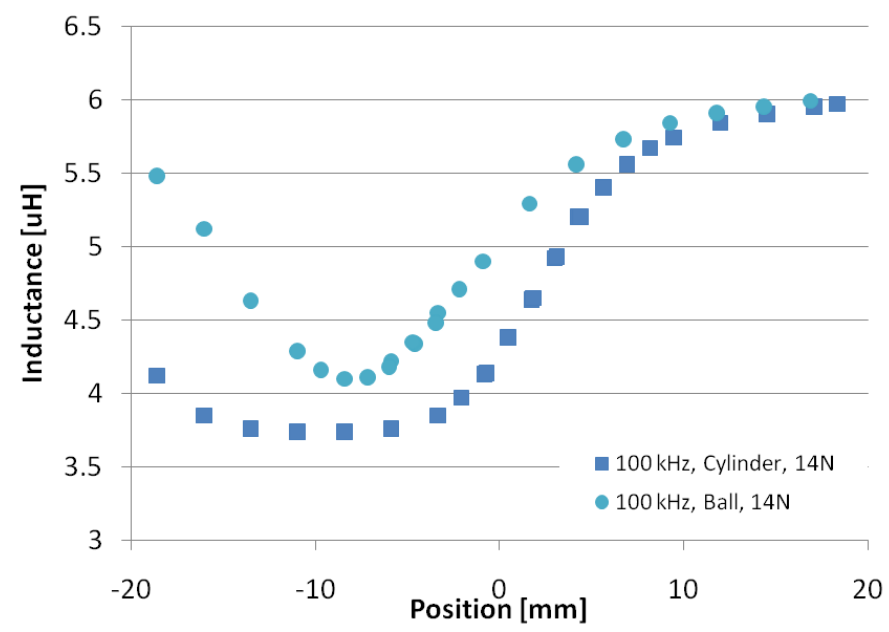

Figure 16. Coil and macron total inductance for cylindrical and spherical macrons, measured as a function of position relative to the coil center. 
synchronized well with the macron passage. A key parameter in coupling efficiency is the mutual inductance between macron and the driver coil (see Eq. 2). As this quantity is difficult to calculate accurately, it was found that the most straight forward (as well as most appropriate) way was to simply measure it. This was done for both the cylindrical and spherical macrons with the results shown in Fig. 16. The coupling was higher for the cylindrical macron, but the spherical macron produced a better force gradient for position and velocity control. As mentioned, it is difficult to properly drive the macron during the startup phase. The energy coupling efficiency, $\eta_{\mathrm{e}}$, is defined as the ratio of the kinetic energy acquired by the macron to the coil magnetic energy. As indicated in Fig. 17, the energy coupling steadily improves for later stages at higher macron velocity where $\eta_{\mathrm{e}}$ approaches the theoretical maximum of $45 \%$ inferred by Eq. (2) and the measured mutual inductance for the prototype coils and cylindrical macrons. Higher efficiencies are achievable with larger coils. The phase II effort will in fact employ larger macrons to increase coupling efficiency. Different macron shapes will also be tested such as a bullet shaped macron that combines the advantages of both the cylinder and spherical macrons considered so far.

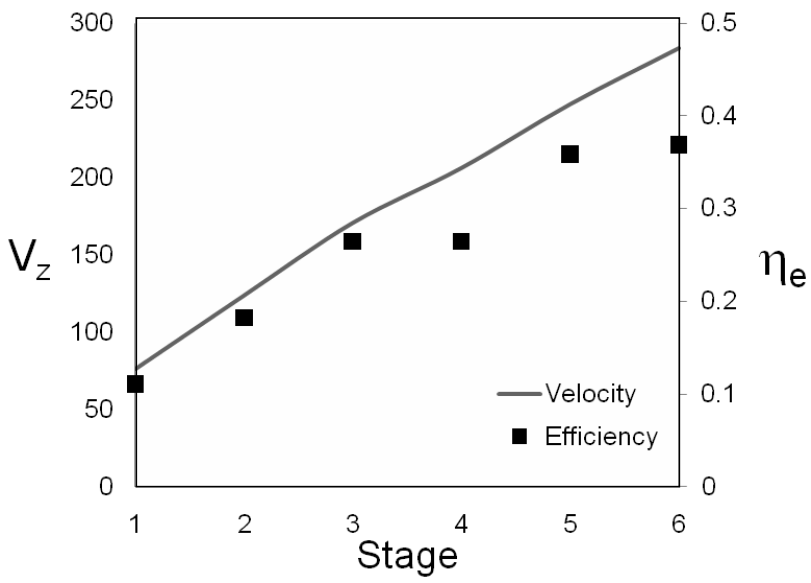

Figure 17. Macron velocity and energy coupling efficiency at each stage.

The phase one plan was to employ more stages of acceleration and reach higher terminal velocities. While there was sufficient switching electronics, driver boards, and materials for the longer launcher, the capacitive energy storage became an issue. The energy storage requirements for higher velocities increase rapidly with velocity. Sufficient energy storage was available in the form of several hundred $1 \mathrm{kV}, 100 \mu \mathrm{F}$ ( $50 \mathrm{~J}$ ) metalized selfhealing capacitors (GA 310DM550). Unfortunately these capacitors have a rapidly decreasing $\mathrm{Q}$ above $5 \mathrm{kHz}$ so that by $20 \mathrm{kHz}$ the Q is less than 5. These capacitors were thus of little use beyond the initial stages. It was possible to obtain surplus IGBT 'snubber' capacitors that operate at very high $\mathrm{Q}$ from several different vendors. A sixteen stage launcher was constructed

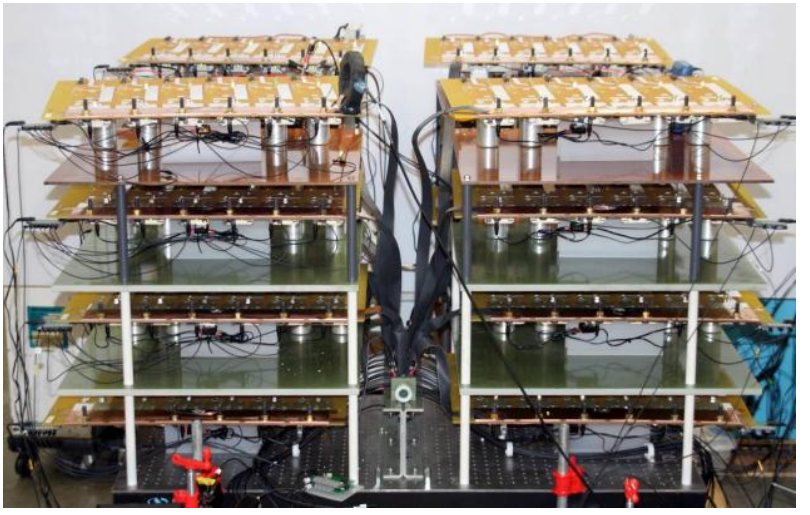

Figure 18. Recently completed 16 stage launcher at MSNW and is now in the process of shakedown and calibration. A picture of the launcher and drivers can be found in Fig. 18. Further discussion of the design and plans for phase II, which will increase the number of stages to bring the macron velocity to that desired for the reactor application, will be found in the work plan section. 


\section{Technical Objectives}

Several key issues have been addressed with the phase I experiments with encouraging results regarding the most critical aspects of the MFL. (1) Both experimental and numerical results indicate that hollow macrons will converge uniformly without any indication of instability. (2) The numerical results show that the macrons can be successfully merged and compressed, even with considerable delay and velocity jitter when compared to what is expected in the final macron accelerator, and observed in the limited experimental testing. (3) Both rotational momentum addition and (4) simultaneous radial and axial convergence was established with macron rings using the 3D ANSYS Multi-physics calculations. (5) In a more severe test than expected for the actual macron liner, a ring of mini-macrons was compressed carrying several 100 kiloamperes of image current. The current rise occurred from a standstill start of the macron ring with only light contact between individual macrons, well before significant merging had occurred. This bodes well for the high currents the macrons must carry at peak compression. Having accomplished these tasks now permits the phase II effort to focus on the most significant issues with regard to both the macron formed liner concept namely the full scale launcher and the efficacy of macron ring liners in compressing the FRC.

The phase II effort will be thus two-pronged. It is critical to test whether the FRC can be compressed to fusion conditions by a macron liner, and it must now be demonstrated experimentally what was observed numerically: that an assembly of macrons can be merged compressing a seed axial magnetic field to the mega-gauss range. To combine these two tasks in one device would thoroughly complicate and jeopardize completion of both tasks. In order to provide sufficient coverage for FRC compression, an axial array of at least four macron rings will need to be constructed, each ring driver being capable of accelerating the array of macrons to a minimum of $2 \mathrm{~km} / \mathrm{sec}(2 \mathrm{~mm} / \mu \mathrm{sec})$ to keep the compression time reasonable. It must be realized that such a system would be close to that desired for the reactor. The energetics of such a large system are well beyond the phase II funding, and the timescale for assembly of the large energy storage and solid state switching arrays would severely limit the time required for operation. It is essential however that a full prototype of one macron accelerator be built and tested. This system can be done in such a way that it can later be broken down to operate up to five accelerators with a modest addition of accelerator energy so that a high energy compression experiment can also be conducted. Even though the macrons will be at somewhat lower velocity $(\sim 1.5 \mathrm{~km} / \mathrm{s})$, there would be sufficient energy to demonstrate merger and compression of the macrons as well as the compression of an axial magnetic field. The five macron ring liner has been modeled in ANSYS Multiphysics, and the results will be discussed in the next section. These two steps comprise the plan to demonstrate and validate the liner production aspect of the MFL fusion system.

The other major effort will be the compression of a merged FRC with a set of four macron rings driven by theta compression coils. This would be a daunting task by itself. What makes it attainable is the relative ease found in driving the mini-macrons in phase I, as well as the fact that it can be done in concert with the Foil Liner Compression experiment already underway at MSNW. The source FRCs and associated diagnostic hardware can be provided by the foil liner test facility when the macron ring compression system is ready for installation. The phase II effort will focus on the construction of the appropriate target chamber, macron rings and driver supplies required for the compression. The macron compression system will be made in manner the permits for it to be swapped in with the minimal impact on the FLC test bed. The initial 
testing will be done with a single macron ring much like as was done with the mini-macron ring in phase I. Here however the coil geometry will be optimized for coupling to the macron ring and of sufficient energy to bring the merging macrons to a radial velocity of $2 \mathrm{~km} / \mathrm{sec}$. This two step progression will be the basic plan for the demonstration and validation of the macron ring compression of the FRC.

Based on these two goals to be accomplished during phase II, one arrives at the following four technical objectives. These are further broken down into several subtasks which are also delineated. The discussion each of the tasks in greater depth will follow in the Work Plan section.

(1) Design and construct the prototype macron launcher, test structure, and target chamber. Operate macron launcher up to target kinetic energy and velocity.

a) Design test bed, launcher coils, insulators, feed plates and cabling system to be employed during testing. Order all long lead items for the MFL test bed.

b) Modify existing solid state switching modules with appropriate capacitor modules. Build new accelerator stages. Construct coils and coil diagnostics.

c) Assemble and test all components at the board level. Design and construct target chamber capable of mounting five separate macron launchers. Fabricate initial macron projectiles.

d) Install all accelerator modules on test bed. Test full system in place.

e) Install a rough vacuum system for macron coil tests. Provide magnetic and visual diagnostics for macron timing and velocity characterization

f) Install control and data acquisition system for bank and macron diagnostic systems.

g) Test the macron launch system to $2 \mathrm{~km} / \mathrm{sec}$.

(2) Design, construct, and assemble a test facility for the theta pinch implosion of a macron ring structure. Generate a uniform macron ring compression to target velocity of $2 \mathrm{~km} / \mathrm{sec}$.

a) Design and assemble test chamber, feed plates, and cabling for the theta pinch driver coil. Design and build optimized macron ring driver coil.

b) Fabricate appropriate macrons for ring and mounting system for test.

c) Install all switch/capacitor modules on test bed. Test full system in place.

d) Install rough vacuum system for macron ring liner tests. Provide magnetic and visual diagnostics for macron ring timing and velocity characterization

e) Install control and data acquisition system for bank and macron diagnostic systems.

f) Test the macron ring liner implosion to $2 \mathrm{~km} / \mathrm{sec}$.

g) Make detailed comparison with numerical predictions

(3) Reconfigure the prototype macron launcher for operation as five individual launchers and mount on suitable structure with merging chamber. Operate the five macron launchers simultaneously to target kinetic energy and velocity with seed axial field. Study magnetic compression.

a) Assemble and test all bank components. Design and construct target chamber capable of mounting five separate macron launchers. Fabricate initial macron projectiles. 
b) Install rough vacuum system for macron merging tests. Provide magnetic and visual diagnostics for macron merging and magnetic field compression characterization

c) Install control and data acquisition for five launcher system.

d) Operate the five macron liner implosion at 1 to $1.5 \mathrm{~km} / \mathrm{sec}$.

e) Make detailed measurements for comparison with numerical predictions

f) Perform numerical studies to understand dynamics of the merging and flux compression.

g) Analyze the tolerance for both jitter and delay in macron motion.

(4) Mount compression chamber with four macron ring structures and associated driver assemblies on the FLC test bed. Compress merged FRCs employing the theta pinch driven implosion of the macron rings. Characterize plasma liner dynamics and evolution of basic FRC parameters during compression.

a) Design and fabricate compression chamber and driver coils. Assemble feed plates, cabling, switches and capacitors for the theta pinch driver coils.

b) Fabricate appropriate macrons and ring mounting system for test.

c) Install compression chamber to FLC vacuum system. Provide magnetic and visual diagnostics for macron ring dynamic behavior and plasma interaction characterization

d) Install all switch/capacitor modules on FLC test bed. Test full system in place.

e) Install control and data acquisition system for macron driver bank and macron diagnostic systems.

f) Test the four macron ring liner implosion to $2 \mathrm{~km} / \mathrm{sec}$.

g) Study macron ring liner compression of the FRC.

\section{Phase II Work Plan}

(1) Design and construct the prototype macron launcher, test structure, and target chamber. Operate macron launcher up to target kinetic energy and velocity.

The design of the macron launcher constructed in phase I was driven mostly by consideration of existing hardware. Each coil of the accelerator was powered by a driver board similar to the one shown in Fig. 19. Each stage employed a driver consisting of 6 parallel high voltage (1700V), high current (4 kA pulsed) Insulated Gate Bipolar Transistor (IGBT) switches. The axial distance between each stage is $1.3 \mathrm{~cm}$ so that the 20 stage accelerator was dwarfed by the driver board arrays needed to power it (see Fig. 18).

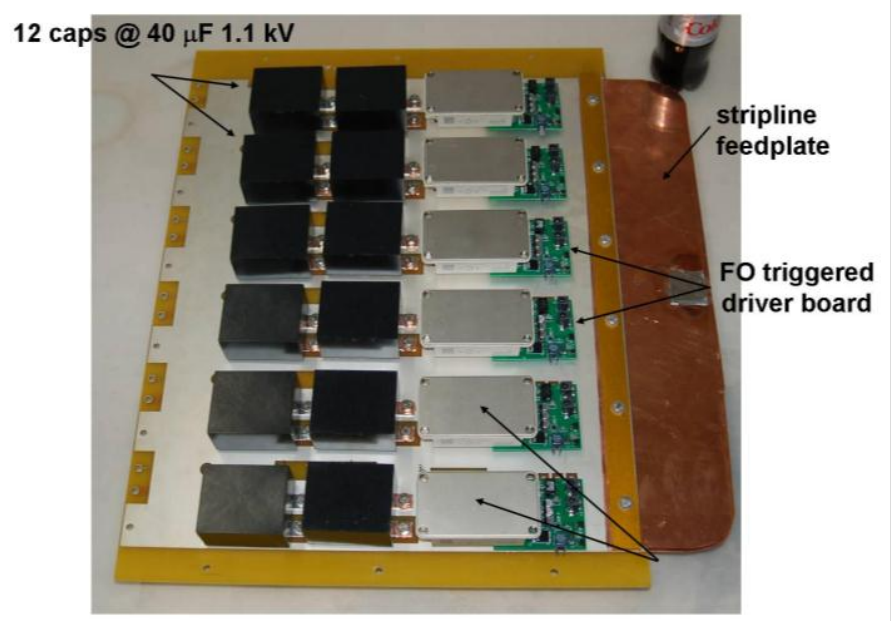

Figure 19. Coil driver to be employed on the phase I macron launcher. 
The driver boards were developed for space application [17] and were designed for minimal driver inductance. As can be seen in Fig. 19, the switching and energy storage are built on a large scale PC board with stripline feedplates. The energy storage in this case was provided by low inductance, high $Q$ poly-foil capacitors. The total stray inductance for the driver at the load is less than $10 \mathrm{nH}$. With stripline connections there is no great penalty for bulky drivers and connection to the 16 stage accelerator was achieved without difficulty (see Fig. 20).

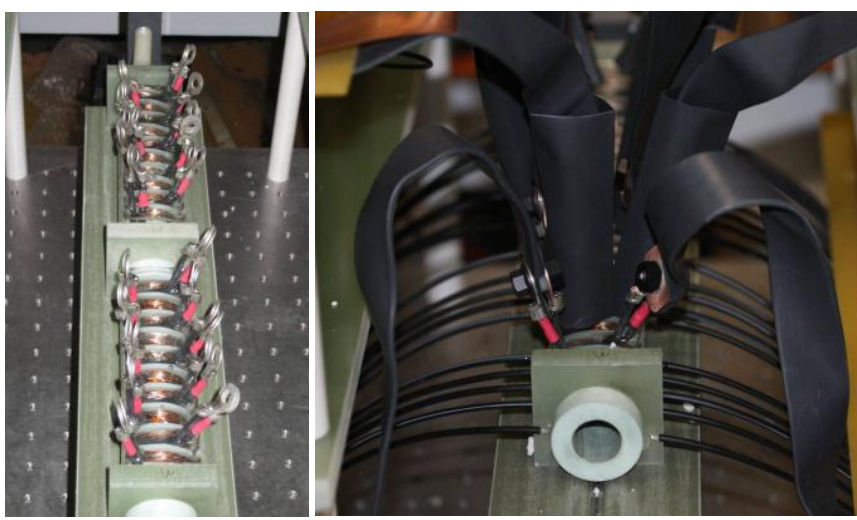

Figure 20. 16 stage accelerator and stripline feeds from array of coil driver boards.

Based on the phase I results, the maximum energy imparted to the macron per stage was roughly $100 \mathrm{~J}$. The limitation comes mainly from the desire to keep the accelerating field to $20 \mathrm{~T}$ and the driver current under $25 \mathrm{kA}$. If one assumed a coupling efficiency of $40 \%$ for the prototype macron launcher, the local energy storage will need to be roughly $250 \mathrm{~J}$ per driver board. As will be discussed, the larger macron to be employed in phase II $(\mathrm{r}=1.5 \mathrm{~cm})$ should provide for greater coupling efficiency. For the initial design a worst case scenario is adopted The ultimate goal is to provide the $2 \mathrm{~g}$ macron with $5 \mathrm{~kJ}$ of directed energy $\left(\mathrm{v}_{\mathrm{f}} \sim 2.2 \mathrm{~km} / \mathrm{s}\right)$. A possible trade-off may be a lower mass macron ( 1 instead of 2 grams), for an increase in terminal velocity $(3 \mathrm{~km} / \mathrm{sec})$.

Critical to maximizing the macron kinetic energy is a basic understanding of the parameters that most significantly affect the energy coupling efficiency of the inductive accelerator. The parametric dependence of the energy transfer to the macron will now be briefly outlined. It is also possible to enhance the energy transfer by providing optimum axial magnetic waveform to the launcher. How this is carried out will also be discussed.

Consider the case where an axial magnetic field is introduced into a flux conserving coil (see Fig. 21). The flux

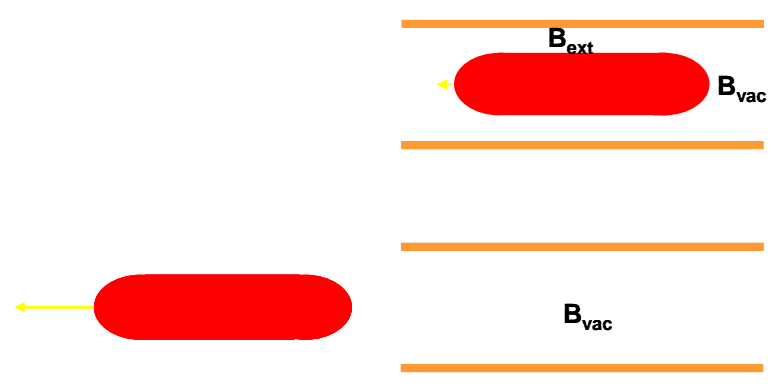

Figure 21. Ejection of a conducting body from a flux conserving coil. (and thus energy) is held constant as the conducting body drifts to the coil edge and is accelerated out of the cylinder by the gradient field there. The final state has the coil empty with a vacuum magnetic field $\mathrm{B}_{\mathrm{vac}}$, and the projectile of mass $M_{p}$ moving away at a velocity $v_{z}$, and kinetic energy $E_{k}=1 / 2 M_{p} v_{z}^{2}$. No energy is added to the system as the projectile is ejected so applying energy conservation from the state before to after ejection results in: 


$$
\frac{B_{\text {ext }}^{2}}{2 \mu_{0}}\left(A_{c}-A_{p}\right) L_{p}-\frac{B_{v a c}^{2}}{2 \mu_{0}} A_{p} L_{p}=\frac{1}{2} M_{p} v_{z}^{2}
$$

From flux conservation:

$$
\mathrm{B}_{\mathrm{ext}}\left(\mathrm{A}_{\mathrm{c}}-\mathrm{A}_{\mathrm{p}}\right)=\mathrm{B}_{\mathrm{vac}} \mathrm{A}_{\mathrm{c}}
$$

where $A_{c}$ and $A_{p}$ are the cross sectional area of the coil and projectile respectively. Equations (11) and (12) together determine the kinetic energy gained by the projectile in terms of the vacuum magnetic energy:

$$
E_{K}=E_{B V} \hat{z}\left(\frac{\hat{x}^{2}}{1-\hat{x}^{2}}\right) \quad \text { where } \quad \hat{z}=\frac{L_{p}}{L_{c}}, \quad \hat{x}=\frac{r_{p}}{r_{c}}
$$

It can be seen that the projectile energy is only a function of the vacuum field and projectile/coil geometry. Not surprisingly, the closer the projectile radius is to the coil radius the better the energy coupling. This dependency is shown graphically in Fig. 22. For a centimeter scale coil, a reasonable maximum value for $\mathrm{x}$ is $\sim 0.9$. For a projectile to coil length ratio of 0.7 , the achievable projectile kinetic energy would be nearly three times the vacuum field energy. This may seem odd, but it merely reflects the fact that the projectile has a profound influence on the circuit as it enters and then exits the coil. On entering the projectile significantly reduces the coil volume. The external circuit momentarily sees a much lower coil inductance and the circuit current (and thus magnetic field) increases considerably. This provides for a much larger magnetic force to act on the projectile as it exits. Another way of thinking of it is that flux conservation requires that the magnetic field, $B_{\text {ext }}$, between the coil and projectile to significantly increase. Flux exclusion from the projectile induces a large current in the coil and equally large counter current in the projectile providing for a large repulsive force.

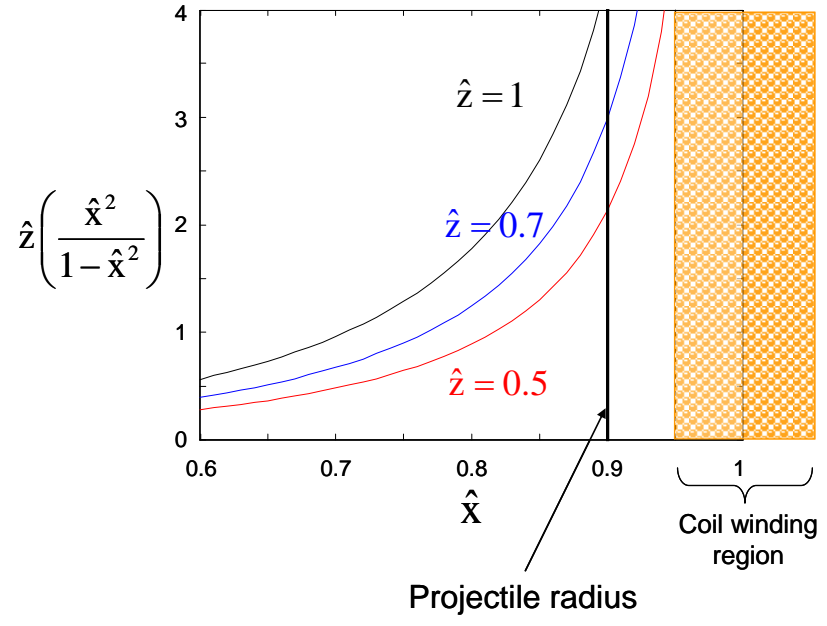

Figure 22. Dependence of energy coupling on coil-projectile radius and length ratios. See Eq. (13).

The issue with smaller macrons and hence coils is the finite thickness required for the coil windings. The effect of a finite coil thickness is to reduce the maximum $\hat{\mathrm{x}}$ achievable. In order to keep the resistive losses low it is necessary to have a sufficient cross-section for the coil. In order to utilize this cross section at the later stages of acceleration, the conductor must be Litz wire. This was done on the initial six stage launcher as well as the current 16 stage launcher. The Litz wire was made from a bundle of 300 minute insulated wires that are grouped and twisted so that the current is evenly distributed 
throughout the cross section at high frequency. Litz wire loses its advantage at megahertz frequencies, but the frequencies required by the MFL are no more than $100 \mathrm{kHz}$ where Litz wire is very effective. Potting the Litz wire in a structural epoxy reinforces the epoxy with a wire matrix that makes for a very strong coil. Similar coils have operated repetitively at magnetic fields of up to $40 \mathrm{~T}$. In the phase I tests, the peak fields were close to $25 \mathrm{~T}$ with no sign of deterioration after hundreds of discharges. The initial coils receive the greatest stresses as they must be powered for the longest time. The final stages will have very little net impulse or heating.

When account is taken for launcher wall thickness, macron dimensional tolerance, and winding thickness, the maximum calculated efficiency was roughly $50 \%$. An efficiency of up to $40 \%$ was obtained for the final stage of the test launcher. While energy recovery makes high efficiency less critical, a lower efficiency requires more accelerator energy storage and length. For a reactor one may not care, but for the phase II effort this translates into dollars and time. The best way to increase efficiency is to simply increase the radial scale. All of the fixed dimensions that limit coupling, such as the coil, wall, and macron clearance now become a smaller fraction of the total and the maximum $\hat{\mathrm{x}}$ that can be achieved increases. The plan is to increase the nominal macron radius from $1 \mathrm{~cm}$ to $1.5 \mathrm{~cm}$. Although the design will conservatively assume $40 \%$ coupling, a coupling efficiency of $60 \%$ is certainly feasible.

The standard operation of the solid state drivers is to produce a full sinusoid period as the capacitor first discharges into the coil followed by current reversal and a recharging of the capacitor at which point the circuit is opened. The full cycle returns all unused energy to the capacitor for the next pulse. With this type of operation, the only loss is the energy transferred to the projectile and a much smaller ohmic loss to the circuit. It is the ability of the macron launcher to be operated in this manner that makes for an extremely efficient liner driver.

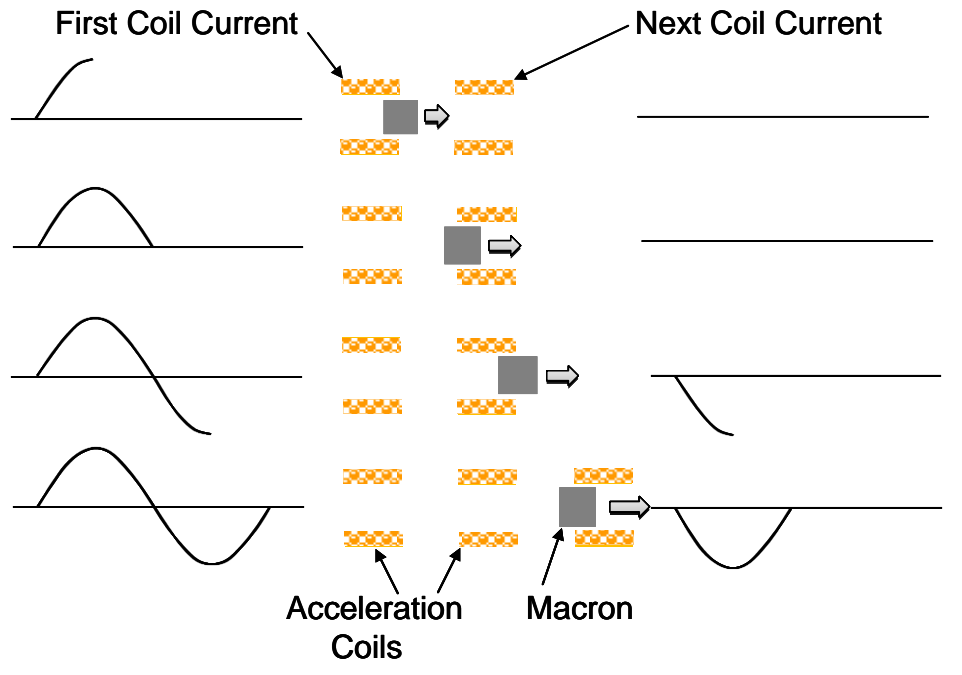

Figure 23. Acceleration methodology with full current waveform. Each coil is pulsed for full cycle. Alternate coils are driven with the opposite polarity, and sequenced to be in phase with the previous coil to maintain maximum field gradient across projectile 
In order to utilize the full cycle of the current waveform, and at the same time significantly improve the stability of the macron with regard to tilt is to sequence the stages such that the accelerating magnetic field direction is reversed with each stage as well. The effective field swing is now much larger in magnitude than what can be obtained with a single pulse in a unidirectional mode. With the proper timing and oscillation frequency, the reversal field of the currently activated coil can be employed to reinforce the field of the next acceleration stage coil (see Fig. 23). Since the inductive force on the macron is through diamagnetic

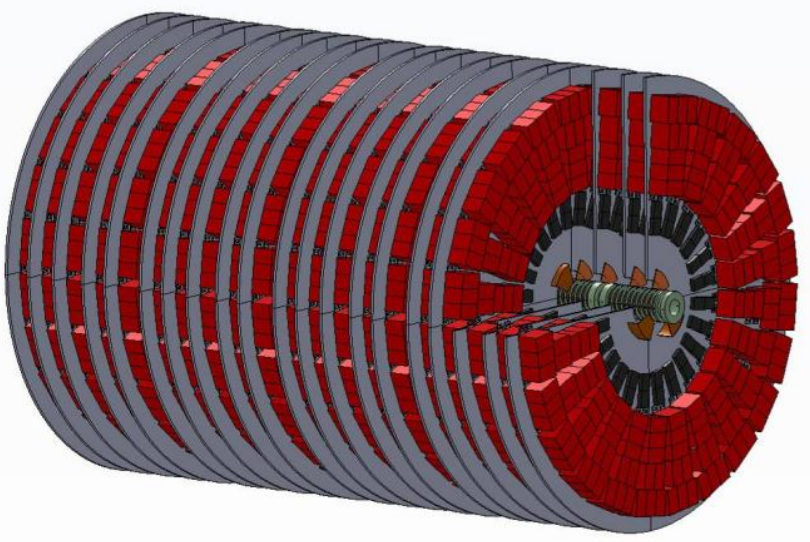

Figure 24. 20 stage section of the prototype macron accelerator to be constructed in phase II currents, the reversal also re-zeros the current induced in the macron. Otherwise, the continuing unidirectional accelerating field would eventually begin to penetrate the macron thereby reducing the drive force. The lack of any net current flowing in the macron also removes the driving force for any tilt-like motion that may occur due to random small motions of the macron.

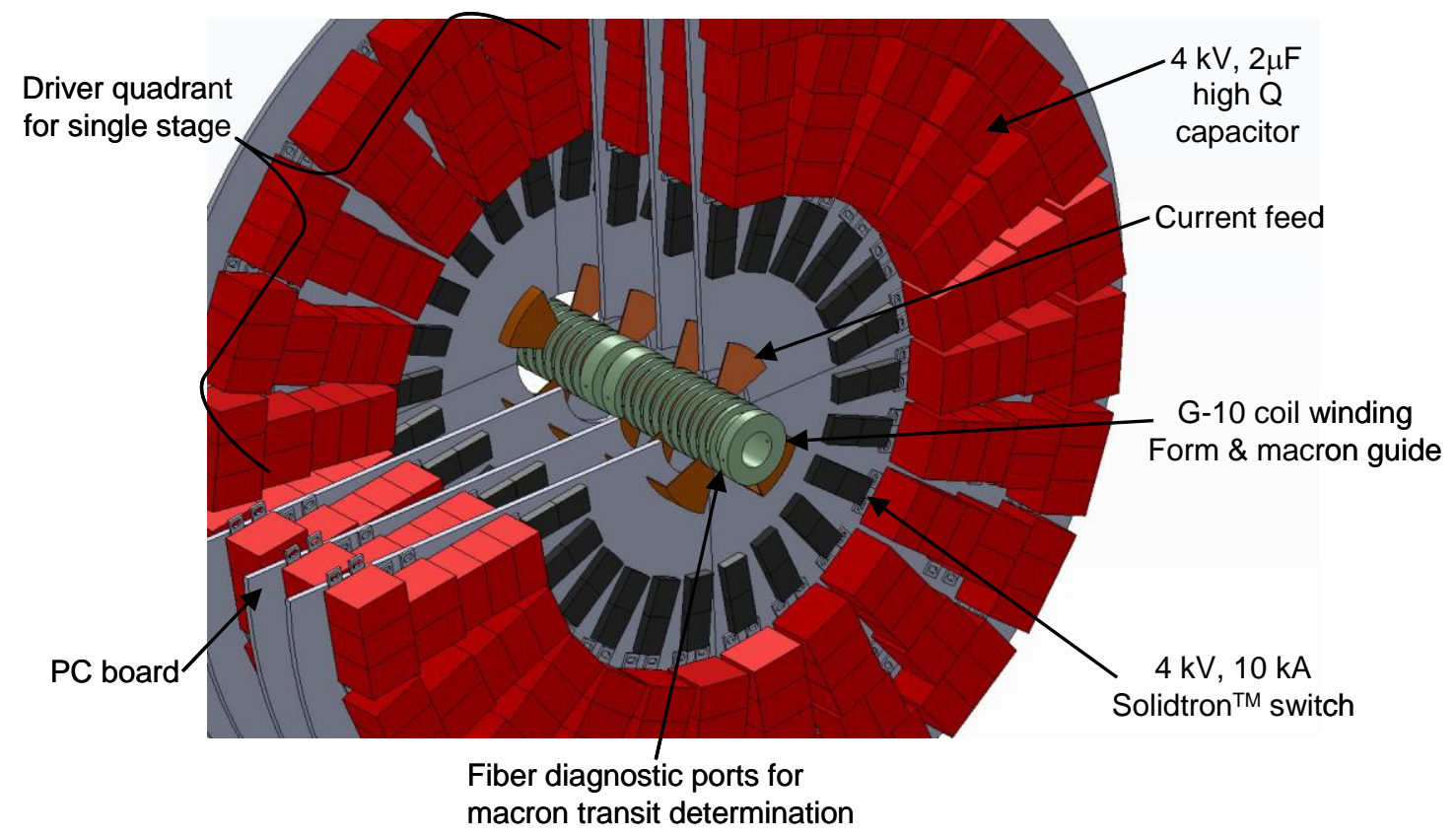

Figure 25. Blowup of cutaway shown in Figure 23.

At $100 \mathrm{~J}$ per stage, the $5 \mathrm{~kJ}$ target energy will require roughly 50 stages. Several optimizations in the design of the driver were explored during phase I. A much smaller and 
higher voltage solid state switch was found in the Solidtron ${ }^{\mathrm{TM}}$ manufactured by Silicon Power. The device is rated at $4 \mathrm{kV} @ 10 \mathrm{kA}$ pulsed. These devices are also surface mountable. Employing arrays of high voltage, high frequency snubber capacitors, all of the driver elements can be mounted on a PC board structure with much less area and depth. To make maximum use of the space around the accelerator, the boards will be annular with each board divided into quadrants each powering a single stage of the accelerator. With each coil stage separated by 1.5 $\mathrm{cm}$, the axial extent of each board will be kept to roughly $5 \mathrm{~cm}$. A CAD drawing of the preliminary design of a 20 section segment of the launcher is shown in Fig. 24. Details of the design are best seen in the blow up of the cutaway section found in Fig. 25.

(2) Design, construct, and assemble a test facility for the theta pinch implosion of a macron ring structure. Generate a uniform macron ring compression to target velocity of $2 \mathrm{~km} / \mathrm{sec}$.

An important aspect of the MFL concept is the behavior of the liner during compression of the FRC. As in a conventional liner, the impact forces involved in merging and compression need to substantially exceed the yield strength of the materials. The hollow macrons readily yield compared to solid materials. In addition, the macron ring liner must not buckle but move inward smoothly, thickening as it is compressed in azimuth and radius. The results from phase I are encouraging in that symmetry was maintained even when the compressive force was inadequate. It certainly should be possible to impart sufficient force to the stationary macron ring with a proper coil and driver design. In this manner the task is no different than that of the strap liners used by Cnare [14] where it was possible to launch foils to velocities of $2.3 \mathrm{~km} / \mathrm{s}$ which had similar mass, as well as radial and axial extent to the macrons to be employed here. These same foils were capable of generating megagauss fields as well.

\section{Magnetic Field Compression of the macron ring liner}

With the use of a theta pinch coil for the driver there is the hidden benefit in that the seed compression field is provided from flux leakage through the liner during the initial stages of acceleration. With the initiation of the $\theta$-pinch current the field rises rapidly in the small radial gap between the external coil and the liner as the liner acts to shunt almost all of the coil inductance. A large driving field is developed, and this external field then diffuses into the cylinder with a characteristic diffusion time given by:

$$
\tau=1 / 2 \mu_{0} r_{L} \delta \sigma_{L}
$$


where $r_{L}$ is the initial (inner) cylinder radius, and $\sigma_{L}$ is its electrical conductivity. The diffusion of the field is then governed by the equation:

$$
\tau \frac{\mathrm{dB}}{\mathrm{dt}}=\mathrm{B}_{\mathrm{ext}}-\mathrm{B}
$$

The dynamics of the liner implosion are then governed by the equation:

$$
M_{L} \frac{d^{2} r}{{d t^{2}}^{2}}=\left(\frac{B^{2}}{2 \mu_{0}}-\frac{B_{e x t}^{2}}{2 \mu_{0}}\right) 2 \pi r
$$

An approximate analytical solution to this equation was obtained as well as a numerical solution with the result being a close match to the results of the Cnare experiments (see Fig. 26). An expression for the peak magnetic field attained at maximum compression was derived. Stated in terms of experimentally relevant quantities, one has

$$
\mathrm{B}_{\mathrm{m}}=\frac{\mu_{0}^{2}}{2 \sqrt{7}} \frac{\mathrm{V}_{\mathrm{c}} \delta \sigma_{\mathrm{L}}}{\mathrm{L}_{\text {tot }}}\left(1+\varepsilon^{2}\right)^{-1 / 2}
$$

where $\mathrm{L}_{\text {tot }}$ is the total driver circuit inductance including stray, and $\mathrm{V}_{\mathrm{c}}$ is the charge voltage on the capacitor bank. For a properly designed driver circuit, the coil inductance will be the dominant inductance. In this case

$$
\mathrm{L}_{\mathrm{tot}} \cong \mathrm{K}_{\mathrm{L}} \mu_{0} \frac{\pi \mathrm{r}_{\mathrm{c}}^{2}}{1_{\mathrm{c}}}
$$

The correction for finite length $\mathrm{K}_{\mathrm{L}}$ can be approximated by $1 / 2\left(1_{\mathrm{c}} / \mathrm{r}_{\mathrm{c}}\right)^{1 / 2}$ for short coils. For $1_{c}=2 r_{c}(\varepsilon \sim 1)$, one has then $L_{\text {tot }}=1 / 4 \sqrt{ } 2 \mu_{0} \pi r_{c}$. Substituting these values into Eq. (17) one has:

$$
\mathrm{B}_{\mathrm{m}}=\frac{\mu_{0}}{\sqrt{7} \pi} \frac{\mathrm{V}_{\mathrm{c}} \delta \sigma_{\mathrm{L}}}{\mathrm{r}_{\mathrm{c}}}
$$

For the aluminum macron liner, using twice the spherical shell thickness for $\delta$, with a bank voltage of $40 \mathrm{kV}$, one has $\mathrm{B}_{\mathrm{m}}=270 \mathrm{~T}$. This is clearly an overestimate in that the coupling to the macron liner was assumed to be perfect and the temperature effect on conductance was not accounted for. A more reliable upper bound can be made from energy conservation

$$
\mathrm{E}_{\mathrm{k}}=\frac{1}{2} \mathrm{M}_{\mathrm{L}} \mathrm{v}_{\mathrm{m}}^{2}=\frac{\Delta \mathrm{B}_{\mathrm{e}}^{2}}{2 \mu_{0}} \pi \mathrm{r}_{0}^{2}
$$

where the change in B is the difference between a vacuum (no liner) case and one with a liner. From the solution to the dynamical equation of motion (Eq. 16) one has

$$
\mathrm{r}_{0} \cong \frac{2}{\mu_{0} \sigma_{\mathrm{L}} \delta}\left(\frac{\mathrm{L}_{\text {tot }}}{\mathrm{V}_{\mathrm{c}}}\right)^{1 / 2}\left[\frac{14 \mathrm{M}_{\mathrm{L}} \mathrm{r}_{\mathrm{L}}^{2}}{\mu_{0} \pi}\left(1+\varepsilon^{2}\right)\right]^{1 / 4}
$$

where the mass per unit length $M_{L}=\rho_{L} \pi r_{L}^{2}=0.12 \mathrm{~kg} / \mathrm{m}$ for our Aluminum macron liner parameters. Plugging in anticipated values one finds $r_{0}=6.2 \mathrm{~mm}$. Using Eq. (20) with a liner kinetic energy of $100 \mathrm{~kJ}\left(\mathrm{E}_{\mathrm{k}}=710 \mathrm{~kJ} / \mathrm{m}\right)$, one has $\mathrm{B}_{\mathrm{m}}=120 \mathrm{~T}$, which consistent with the Cnare experimental results. 
With the liner radius and field determined, the flux that has diffused inside the liner is 14.5 $\mathrm{mWb}$. Recall that the FRC poloidal flux $\varphi_{\mathrm{p}} \sim 1.5 \mathrm{mWb}$. The FRC equilibrium radius at maximum compression can be related to liner radius by noting that $\varphi_{\mathrm{p}} \cong \mathrm{x}_{\mathrm{s}}{ }^{3} \mathrm{r}_{\mathrm{L}}^{2} \mathrm{~B}_{\mathrm{m}}$. This implies that $\mathrm{x}_{\mathrm{s}}=0.69$ with $\mathrm{r}_{\mathrm{s}}=4.2 \mathrm{~mm}$. The radial compression ratio is $\mathrm{X} \sim 1 / 12$. With no active axial compression, the FRC equilibrium length scales as $(\mathrm{X})^{0.4} \sim 0.37$. The final result is a compression in volume by nearly a factor of 400. From 2D MHD analysis of $\theta$-pinch liner compression of a plasma liner, a significant additional axial compression occurs as a consequence of the presence of the FRC in only the central region of the liner.

The driver coil will typically be no wider than the macron $(\sim 2 \mathrm{~cm})$. To obtain optimum drive, Eqs. (17) and (19) indicate that a single turn coil at the maximum voltage with the minimum stray inductance at the smallest radius is optimum. The radius of the coil will be as small as practical and still maintain the macron ring initial radius a macron diameter outside the FRC chamber inner radius of $5.0 \mathrm{~cm}$. The nominal radius will be $7.5 \mathrm{~cm}$ similar to Cnare's coil radius of $7+\mathrm{cm}$. The voltage will be $40 \mathrm{kV}$ which is twice what Cnare applied. An assessment of the minimization of stray inductance will now be given.

A major difference between the simple foil liner and the macrons is the azimuthal gaps that must be filled with flux thereby increasing the effective stray inductance. Even with this, the stray will be a small part of the total inductance. Without the liner, the inductance of the $\theta$-pinch coil for a coil with a length to diameter ratio of unity is given by the following:

$$
\mathrm{L}_{\mathrm{C}}=\frac{\sqrt{2}}{4} \mu_{0} \pi \mathrm{r}_{\mathrm{C}}=105 \mathrm{nH},
$$

where a coil radius of $7.5 \mathrm{~cm}$ was assumed. The macron ring liner acts like the secondary of a transformer with the coil being the primary. As mentioned, there are two effects that keep the efficiency of coupling at less than unity. There is the transformer coupling coefficient which reflects basically the difference in area between the liner and the coil. It is assumed that the coil will consist of a thick conducting strap affixed to the insulating vacuum wall. The short, subinertial timescale for the presence of a large field acting on this coil considerably reduces the reactive energy impulse delivered to the coil. By tying it firmly to an Alumina vacuum tube wall the coil motion is greatly restricted and the coupling to the liner maximized. An alumina wall of $5 \mathrm{~mm}$ thickness will provide sufficient strength to withstand both the coil rebound and any possible liner fragment forces.

The second source of stray inductance is associated with the coil driver. It is planned to use high energy density capacitors for the compression bank. An estimate of the coupling will now be discussed. The stray inductance from each capacitor/switch module can be readily assessed. There is $\sim 40 \mathrm{nH}$ associated with the capacitor and radial header. A "piggy back" crowbar switch will be employed with these capacitors. A good crowbar is valuable as the risetime of the current can be shorter than the inertial time for liner motion. The inductance associated with the switch is $~ 25 \mathrm{nH}$. Eight 3.5 meter long RG213 cables will be used to connect the cap/switch to the coil feed plates for a total of $100 \mathrm{nH}$ of cable inductance. The total inductance per cap/switch assembly is thus $165 \mathrm{nH}$. With 4 capacitor/switch assemblies per macron ring driver coil this yields a total effective inductance of roughly $40 \mathrm{nH}$. The bank inductance of $40 \mathrm{nH}$ is roughly 
twice that of the gap. One can think of this additional inductance as increasing the effective coil radius $r_{\mathrm{ce}} \cong 9 \mathrm{~cm}$.

With the initial discharge of the compression bank, it is only the total stray inductance $\mathrm{L}_{\mathrm{s}}$ $60 \mathrm{nH}$ that will be limiting the current rise. The quarter cycle time $\tau_{1 / 4}=1 / 2 \pi\left(\mathrm{L}_{\mathrm{s}} \mathrm{C}_{\mathrm{b}}\right)^{1 / 2}=3.2 \mu \mathrm{sec}$. At the full voltage of $40 \mathrm{kV}$, the peak current $\mathrm{I}_{\mathrm{m}}=\left(\mathrm{C}_{\mathrm{b}} / \mathrm{L}_{\mathrm{s}}\right)^{1 / 2} \mathrm{~V}_{\mathrm{b}}=1.3 \mathrm{MA}$. The duration of the large currents (and external gap field $\mathrm{B}_{\mathrm{ex}}$ ) is set by the inertial time for liner movement inward. As long as this is on the order of the current risetime or longer, good coupling can be achieved. Given the fact that the maximum velocity attained by the liner is $2.5 \mathrm{~mm} / \mu \mathrm{sec}$, and that the accelerating force $\propto \mathrm{I}^{2}$, it is a good approximation that the liner will not have moved significantly during the current rise.

An estimate of the maximum liner drive efficiency can now be made with a few additional simplifications. It will be assumed that resistive losses in the coil and macron liner can be ignored, as well as the magnetic energy inside the liner at time of maximum velocity. An ideal crowbar is assumed at current peak so that the magnetic flux and energy inside the effective gap remain constant throughout the liner acceleration to peak velocity, and that this energy is equal to the bank energy, $\mathrm{E}_{\mathrm{CB}}$. In other words:

$$
\mathrm{E}_{\mathrm{CB}}=\frac{\mathrm{B}_{\mathrm{ext}} \varphi_{\mathrm{ext}}}{2 \mu_{0}}=\frac{\mathrm{B} \varphi_{\mathrm{ext}}}{2 \mu_{0}}+\mathrm{E}_{\mathrm{K}}
$$

where all terms are in units of energy/length, and $\varphi_{\mathrm{ext}}=\pi\left(\mathrm{r}_{\mathrm{ce}}{ }^{2}-\mathrm{r}_{\mathrm{L}}{ }^{2}\right) \mathrm{B}_{\mathrm{ext}}$. By flux conservation, the magnetic field behind the liner at small radius, $\mathrm{B} \sim \mathrm{B}_{\mathrm{ext}}\left[1-\left(\mathrm{r}_{\mathrm{L}} / \mathrm{r}_{\mathrm{ce}}\right)^{2}\right]$. Substituting into Eq. (23) one has for the energy coupling efficiency $\eta_{\mathrm{c}}$ :

$$
\eta_{\mathrm{c}}=\frac{\mathrm{E}_{\mathrm{K}}}{\mathrm{E}_{\mathrm{CB}}}=\left(\frac{\mathrm{r}_{\mathrm{L}}}{\mathrm{r}_{\mathrm{ce}}}\right)^{2}=\left(\frac{7}{9}\right)^{2}=0.60 .
$$

In deriving this efficiency flux diffusion and Ohmic losses have been ignored, but it seems reasonable that an efficiency on the order of $50 \%$ is not an unreasonable goal. For the driver just described, this represents a macron ring liner kinetic energy of $27 \mathrm{~kJ}$. For a $6 \mathrm{~g}$ total ring mass, this corresponds to an inward ring velocity of $3 \mathrm{~km} / \mathrm{s}$.

It will be necessary to perform the foil implosion tests with the liner in vacuum as in the phase I tests. Even though atmosphere pressure is negligible compare to the magnetic pressures expected here, it was noticed by Cnare that when the foil implosion experiment was performed in an atmospheric environment, an air shock was formed ahead of the foil. This shock destroyed the magnetic probe used to measure the field inside the liner before foil impact. He thus performed most of the flux compression experiments in a vacuum of roughly $1 \mathrm{mTorr}$. Since a good vacuum is not required, this should not be a significant issue. The system must eventually operate in a good vacuum in any case.

By this point in liner testing the ANSYS Multiphysics numerical liner calculation will have been upgraded to include magnetic field compression for comparison with the diagnostic output 
from the tests. Conventional B dot probes and backlit end-on framing pictures will be the mainstay for diagnosing gross liner behavior as it was in the phase I experiments.

(3) Reconfigure the prototype macron launcher for operation as five individual launchers and mount on suitable structure with merging chamber. Operate the five macron launchers simultaneously to target kinetic energy and velocity with seed axial field. Study magnetic compression.

With the completion of the full power test with the 50-stage macron launcher, the driver boards will be rearranged and augmented to drive five separate launchers with 15 stages each. The macron energy per launcher will be roughly $1.5 \mathrm{~kJ}$. With a $2 \mathrm{~g}$ macron this will yield a terminal velocity of $1200 \mathrm{~m} / \mathrm{s}$. A one gram liner has a corresponding exit velocity of $1700 \mathrm{~m} / \mathrm{s}$. Both should be sufficient to examine flux compression with a macron formed liner. To test the merger and compression dynamics a ANSYS Multiphysics calculation was conducted. Five macron spheres of $3 \mathrm{~cm}$ diameter, 2 gram mass, radially converging at $1.6 \mathrm{~km} / \mathrm{s}$ was conducted. The result is shown in Fig. 27. It is interesting to note that the start of the same buckling observed in the phase I experiment is seen in the cross sectional view (see last frame). However here the macrons still have over $95 \%$ of their original radial velocity. The calculation is halted due to contact at the origin. When an axial magnetic field is present it is expected to be compressed to megagauss fields and eventually halt the converging macrons prior to convergence on axis. The ANSYS Multiphysics package is fully capable of solution with such a magnetic field present, but there wasn't sufficient time during phase I to incorporate it. This will certainly be done in phase II effort.
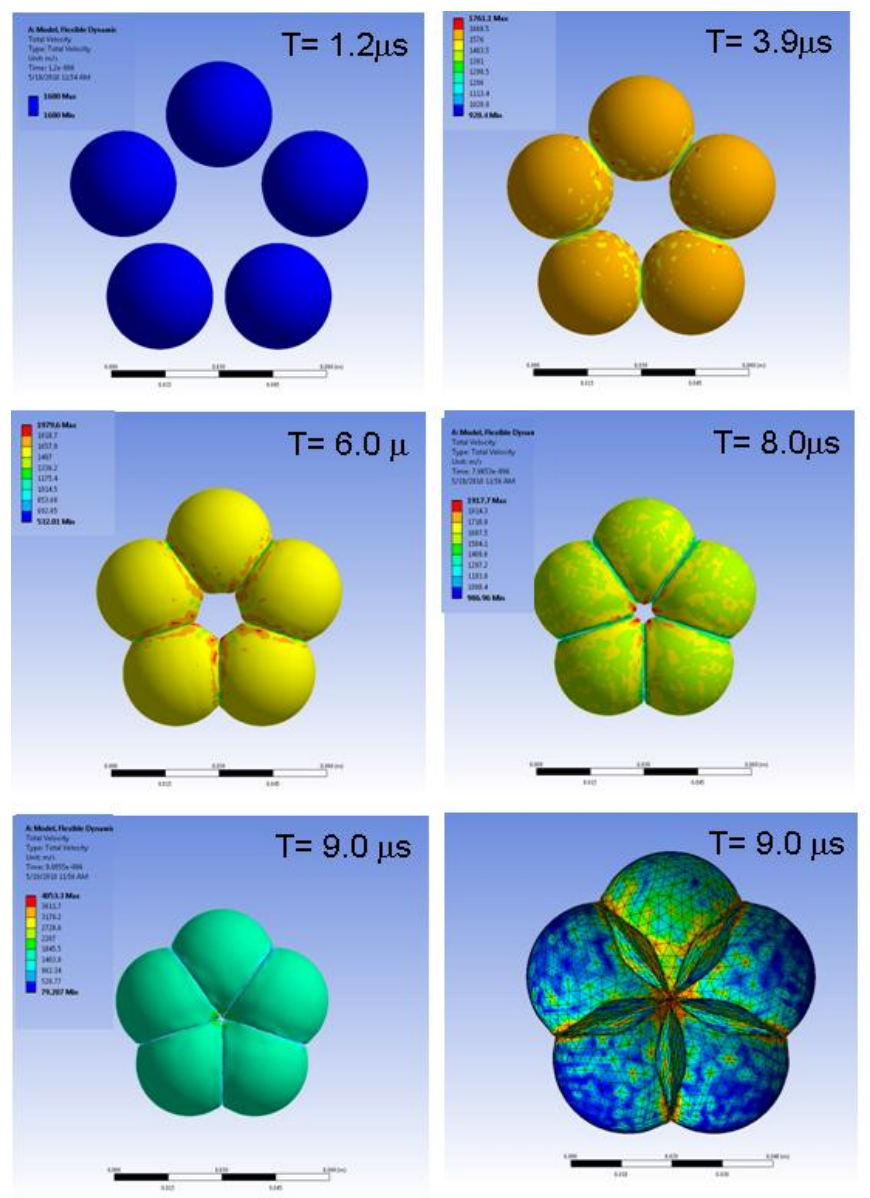

Figure 27. ANSYS Multiphysics calculation for 5 sphere merging and compression experiment. Last frame shows cross section and numerical grid.

\section{Five Macron Merging Experiment}

Each accelerator stage driver board will need to be "retuned" for its new role in the foreshortened launchers. There is a bit of work involved here. For an efficient and uniform acceleration, the risetime of each coil needs to be adjusted for the speed that the projectile has attained up to that point. It is universally true that it is optimum to have all coils be operated at the maximum voltage. The capacitance is also fixed as the stored energy per stage is roughly the same. For a fixed driver capacitance, the oscillation frequency is accomplished by adjusting the 
coil inductance by modifying the number of turns. The situation is somewhat complicated by the shorting effect that the projectile has during its brief transit. This must be taken into account in the final coil design, but for the zero order analysis considered here the inductance will be that of the coil alone. Using the coil parameters from the phase I studies:

$$
\mathrm{L}_{\mathrm{tot}}=\mathrm{K}_{\mathrm{L}} \mu_{0} \frac{\mathrm{n}^{2} \pi \mathrm{r}_{\mathrm{c}}^{2}}{1_{\mathrm{c}}} \cong \frac{1}{2} \mu_{0} \pi \mathrm{n}^{2} \frac{\mathrm{r}_{\mathrm{c}}^{3 / 2}}{1_{\mathrm{c}}^{1 / 2}}=14 \mathrm{n}^{2}(\mathrm{nH}),
$$

where $\mathrm{n}$ is the number of turns, and the finite coil correction $\mathrm{K}_{\mathrm{L}}$ can be approximated for small elongation coils as $\mathrm{K}_{\mathrm{L}} \sim 1 / 2\left(\mathrm{l}_{\mathrm{C}} / \mathrm{r}_{\mathrm{C}}\right)^{1 / 2}$. It is worth characterizing the desired range of inductances from the starting coil to the last coil of the accelerator. The magnetic field swing that that can be achieved between each coil is given by roughly twice the peak field corresponding to what can be provided by the capacitive energy supplied to the coil by the driver. One has for the field created by the fast bank using the nominal coil dimensions:

$$
\frac{1}{2} \mathrm{CV}^{2}=200 \sim \frac{\mathrm{B}_{\mathrm{vac}}^{2}}{2 \mu_{0}} \mathrm{Vol} \Rightarrow \mathrm{B}_{\mathrm{vac}}=8 \mathrm{~T}
$$

For the first coil then the appropriate coil risetime is:

$$
\tau_{1 / 4} \sim \frac{1_{c}}{\mathrm{v}_{0}}=\frac{0.02}{500}=40 \mu \mathrm{sec} \Rightarrow \mathrm{V}_{\mathrm{c}}=\omega \mathrm{A}_{\mathrm{c}} \mathrm{B}_{\mathrm{vac}}=100 \mathrm{~V}
$$

The loop voltage $\mathrm{V}_{\mathrm{c}}$ inferred for the last coil at the terminal velocity of $1.5 \mathrm{~km} / \mathrm{s}$ would be roughly $600 \mathrm{~V}$. This would permit at least a two turn coil, and which from Eq. (25) would raise the coil inductance to $56 \mathrm{nH}$. With the capability of apply a much higher voltage, a multi turn coil can be employed to right to the last coil. The coil inductance will always be considerably more than the driver board stray inductance thus assuring good energy transfer to the coil even with a macron present.

A suitable chamber for the macron compression experiments must accommodate the occasional macron misfire. Each entry port will thus have a port on the opposite side to act as a safety dump. It will be necessary to use such a port for this purpose as the various launchers are each calibrated during testing. A

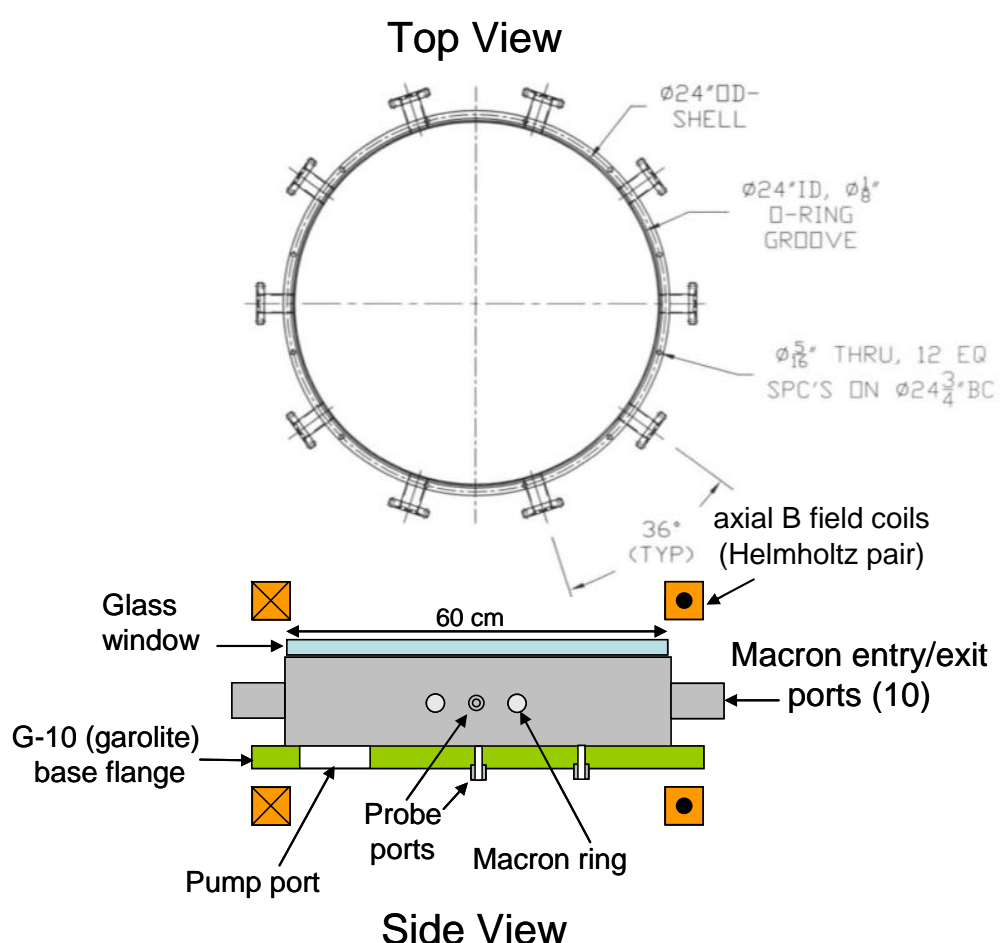

Figure 28. Schematic of the proposed 5 macron merging and compression chamber 
schematic of the chamber is illustrated in Fig. 28.

Conventional B dot probes and backlit side-on framing pictures will be employed to obtain information on gross macron behavior. Interruption of a collimated diode light beam can also be used to relate positional information and velocity along all stages of the launchers. Several 16 channel, 12 bit, $20 \mathrm{MHz}$ digitizers are available to record both the B dot and light signals. Final velocity, position, and arrival time of the macrons can also be obtained by these same diagnostics placed sufficiently downstream of the macron projectile inside the merging chamber. The sequencing of the coils will be initially accomplished by an array of timing generators with $100 \mathrm{MHz}(10 \mathrm{~ns})$ timing accuracy. This should be more than adequate. The motion of the macron is slow enough that it should be possible to do positional feedback on the trigger initiation to assure simultaneous arrival at the merging point. Such a feedback system would also allow for the adjustment and trimming of the macron velocity at exit. This would provide for a powerful technique to minimize arrival jitter of the macrons if needed. The evidence to date from the phase I results is that the accelerator waveform itself creates a magnetic well that synchronizes the macron with it as long as random perturbations that would effect its motion are not too large.

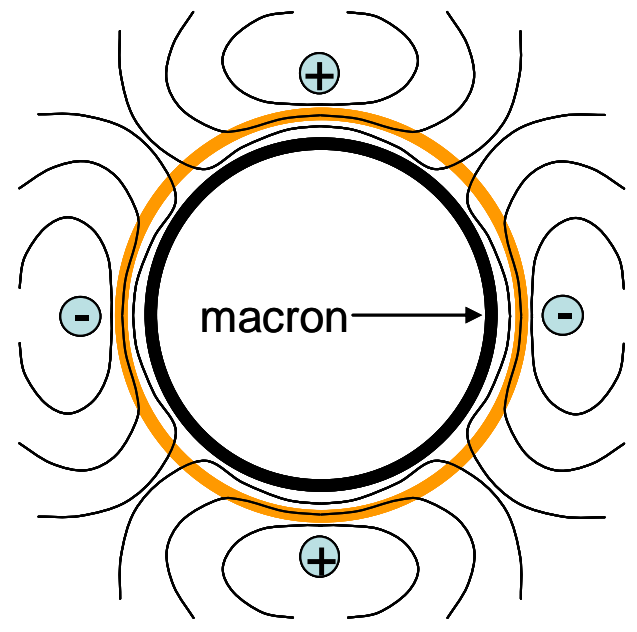

Figure 29. Cross sectional sketch of how quadrupole field coils could be employed for stabilized macron motion.

One other modification that may need to be implemented during the phase II tests would be aimed at addressing the stability of the macron as it propagates down the accelerator. The higher edge field of the coils, as well as some axial field penetration into the macron will help keep the macron centered and stable. If however it is discovered that an unacceptable wobble is occurring, it is relatively straight forward to suppress this motion with a multipole barrier field. This field is transverse to the axial field and can thus be applied by conductors outside the field coils. This would take the form of an azimuthal array of axial conductors. The simplest set would be that of a quadrupole as depicted in Fig. 29. The current requirements would be minimal. The wire could also be pitched helically to apply a rotational torque if additional gyroscopic stability is desired [18]. This barrier field would then act very much like rifling does in a conventional gun barrel.

(4) Mount compression chamber with four macron ring structures and associated driver assemblies on the FLC test bed. Compress merged FRCs employing the theta pinch driven implosion of the macron rings. Characterize plasma liner dynamics and evolution of basic FRC parameters during compression

\section{FRC Compression with Four Macron Ring Liners}

The major task of the first year was to design a coil that could operate at a high energy transfer rate and deliver the requisite energy to the macron ring liner. The desire was to have the ring achieve an inward velocity of $2 \mathrm{~km} / \mathrm{s}(2 \mathrm{~mm} / \mu \mathrm{sec})$. At this speed the compression of a $4 \mathrm{~cm}$ 
radius FRC could be accomplished in $20 \mu \mathrm{sec}$. The plan is to start with much larger FRC formation chambers $\left(r_{c}=10 \mathrm{~cm}\right)$ and produce two FRCs with a much longer lifetime $(\sim 100$ $\mu \mathrm{sec})$ than what would appear necessary for a liner experiment. While this may seem to be overkill, it must be remembered that the FRC lifetime is a strong function of $r\left(\tau \sim r^{2.1}\right)$. While this is compensated somewhat by the observed FRC density scaling $\left(\tau \sim \mathrm{n}^{0.6} \sim \mathrm{r}^{(-2.4) 0.6}\right)$, the overall FRC lifetime will decrease as it is compressed $\left(\tau \sim r^{0.66}\right)$. For a ten to one radial compression the FRC lifetime will decrease by a factor 5. Since the reduction occurs during the compression, there should be more than adequate FRC life even if the compression speed were somewhat slower.

The desire is to keep the energy involved in the four ring liner implosion to a minimum so as

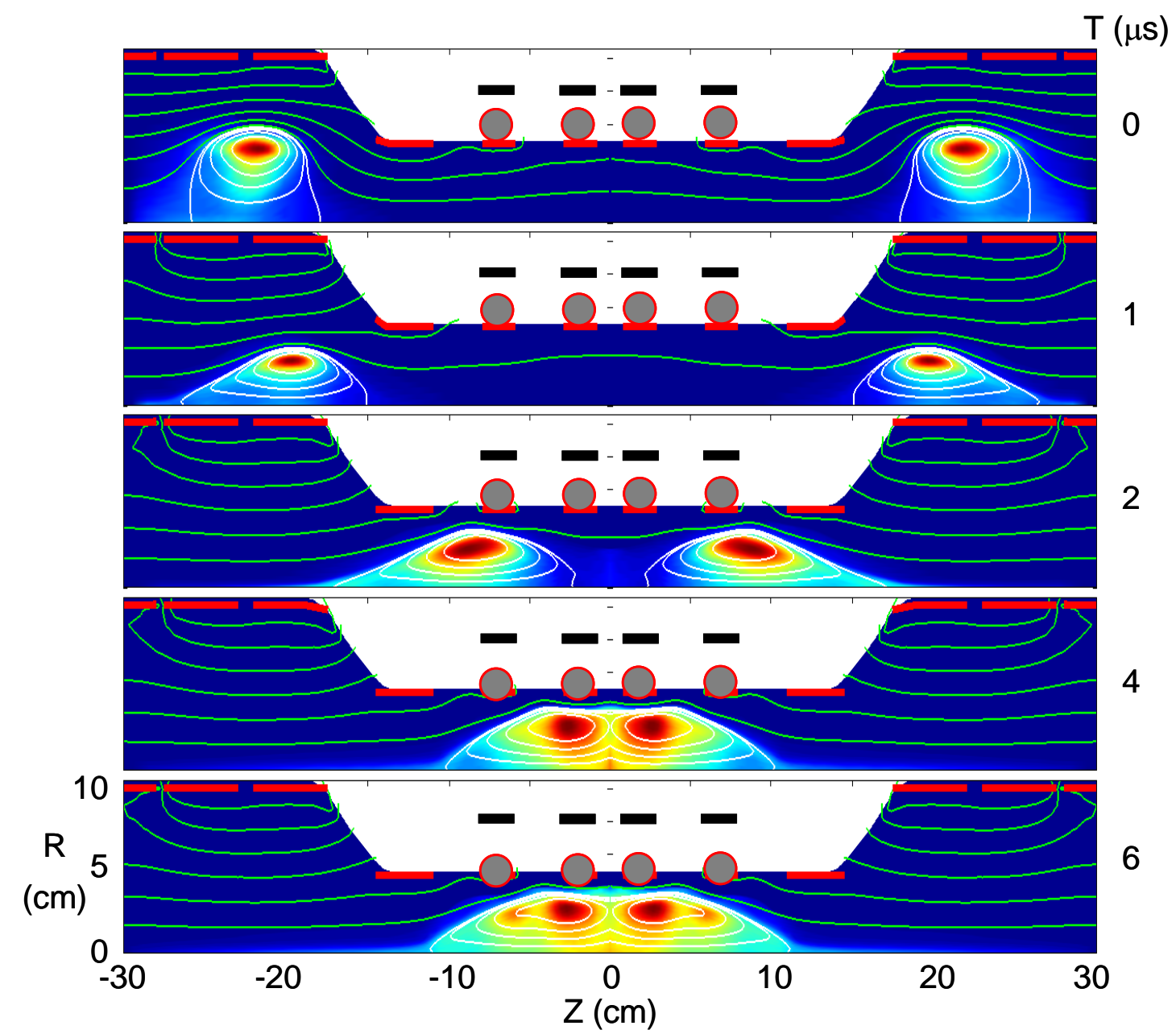

Fig. 30. Resistive 2D MHD calculation employing dynamic FRC formation sequence on the Foil Liner Compression test bed. The macron ring liner driver coils are indicated in black and the macron rings comprised of twenty, $2 \mathrm{~cm}$ diameter, hollow Aluminum spheres are shown in red with a grey interior. The drivers and ring liners are for illustrative purposes only as they were not part of the calculation. The radial change in the macrons indicates the position of the ring moving at $2 \mathrm{~km} / \mathrm{s}$ during FRC formation. 
to limit collateral damage. With each ring having roughly $26 \mathrm{~kJ}$ of kinetic energy imparted to it by the driver the total is $100 \mathrm{~kJ}$, which should be manageable. It is important to have the ability to modify the compression profile. This could be done by varying the position of the macron rings or possibly having various liner segments have different masses, speeds and most significantly, timings. A decision on the initial configurations and timings will be made after the completion of task one and more detailed MHD modeling and calculations. The decision to employ thyratron switches for the drivers that have been used for the FRC formation in the past assure that the timing option to gain more control of the compression as well as maintain the radial pressure on the ring liner and thus increasing the time for liner acceleration.

The resistive 2D MHD numerical calculation was carried out for the target MFL parameters using the Moqui code, and is shown in Fig. 30. The FRC sources and translation chamber were based on the current design for the FLC test bed. The coils used in the calculation are shown in red. The confinement coils were shortened to be the same width as the macrons to give an idea of the magnetic field profile as the FRCs are merged inside the macron rings. Of course there is no motion of these coils in the calculation. Considerable work has been done with a similar code, MACH2 (Multi-block Arbitrary Coordinate Hydromagnetic 2D) to incorporate the solid liner behavior for the MTF experiment [21]. This code is now being modified at the UW to include a theta pinch driver to study the effect of its motion on compressing the FRC as part of the Foil Liner Compression study at MSNW. It is not clear at this point if the code can accommodate an array of short rings as was done in the Moqui calculations above, but this possibility will certainly be investigated.

The magnetic field in the compression section of the calculation shown in Fig. 30 was kept purposely low $(\sim 0.2 \mathrm{~T}$ vacuum $\Leftrightarrow 0.7 \mathrm{~T}$ with $\mathrm{FRC}$ insertion $)$ as there will be little need to have any significant flux with the theta pinch driven macron rings. The vacuum field from the macron driver coils alone will be on the order of $8 \mathrm{~T}$. While the array of rings will keep the greater part of this driver flux out of the inner region during compression, a significant amount will penetrate and maintain, or even increase the FRC magnetic insulation from the rings. The amount of flux leakage will be characterized in Task one studies so that the proper amount of bias field will be present during FRC merging.

The FRC behavior will be determined by the suite of diagnostics being developed for the FLC experiments. These include cross tube visible HeNe based interferometry and magnetic loop arrays. An advantage of the $\theta$-pinch driver method is that the end access is unrestricted. The FRC density can be resolved by the use of end-on interferometry, and end-on imaging in both visible and soft $\mathrm{x}$-rays will provide invaluable information on the FRC equilibrium and stability In addition to these key diagnostics, filtered $\mathrm{x}$-ray detectors will be used to obtain information about the electron temperature evolution. Other end-on diagnostics include bolometry and Doppler spectroscopy. There will also be the usual array of diamagnetic loop pairs. The difference in signal from the vacuum case due to the presence of the FRC maybe more difficult to interpret due to the much larger effect of the liner dynamics. Comparison with the MHD liner codes will be critical in interpreting signal from this diagnostic. In addition to the Doppler diagnostic, two calibrated neutron detectors will be employed for further confirmation of fusion conditions.

The efficiency of the transfer of the macron ring liner energy to the FRC should be high. If one assumes that only $50 \%$ is coupled this represents an increase in the FRC internal energy of $50 \mathrm{~kJ}$. The internal energy of the FRC is given by the following expression: 


$$
\mathrm{E}_{\text {int }}=\mathrm{E}_{\mathrm{th}}+\mathrm{E}_{\mathrm{B}}=\frac{5}{2} \mathrm{Nk}\left(\mathrm{T}_{\mathrm{i}}+\mathrm{T}_{\mathrm{e}}\right)
$$

Where $\mathrm{N}$ is the Deuterium particle inventory. For the calculation of Fig. 30, the particle inventory, $\mathrm{N}=6 \times 10^{18}$. This would imply $\mathrm{T}_{\mathrm{e}}=\mathrm{T}_{\mathrm{i}}=10 \mathrm{keV}$. This temperature increase should reflect an adiabatic scaling, i.e. $\left(\mathrm{T}_{\mathrm{f}} / \mathrm{T}_{\mathrm{i}}\right)=\left(\mathrm{B}_{\mathrm{f}} / \mathrm{B}_{\mathrm{i}}\right)^{4 / 5}$. With an initial to final total temperature ratio of $20 \mathrm{keV} / 0.25 \mathrm{keV}$, and an initial axial confining field $\mathrm{B}_{\mathrm{i}}=0.7 \mathrm{~T}$ implies a final magnetic field of $167 \mathrm{~T}$ (1.7 megagauss). The adiabatic scaling for the density $\left(\mathrm{n}_{\mathrm{f}} / \mathrm{n}_{\mathrm{i}}\right)=\left(\mathrm{B}_{\mathrm{f}} / \mathrm{B}_{\mathrm{i}}\right)^{6 / 5}$ yields a peak final density of $5.6 \times 10^{23} \mathrm{~m}^{-3}$. Getting anywhere close to these results will make for a very exciting conclusion to the phase II effort. 


\section{Performance Schedule}

The performance schedule for the four main tasks and the many subtasks listed in the Technical Objectives (section IV) and detailed in the Work Plan (section V) can be found in the Gantt chart given in Fig. 31. Each subtask for each major task is listed by number and letter corresponding to the breakdown given in the Technical Objectives section. The gantt chart specifies the estimated time and duration for each task. The labor allocations required for these tasks is summarized in the labor allocation section of the budget (parts A and B) and the materials and supplies for each task can be found in the budget details.

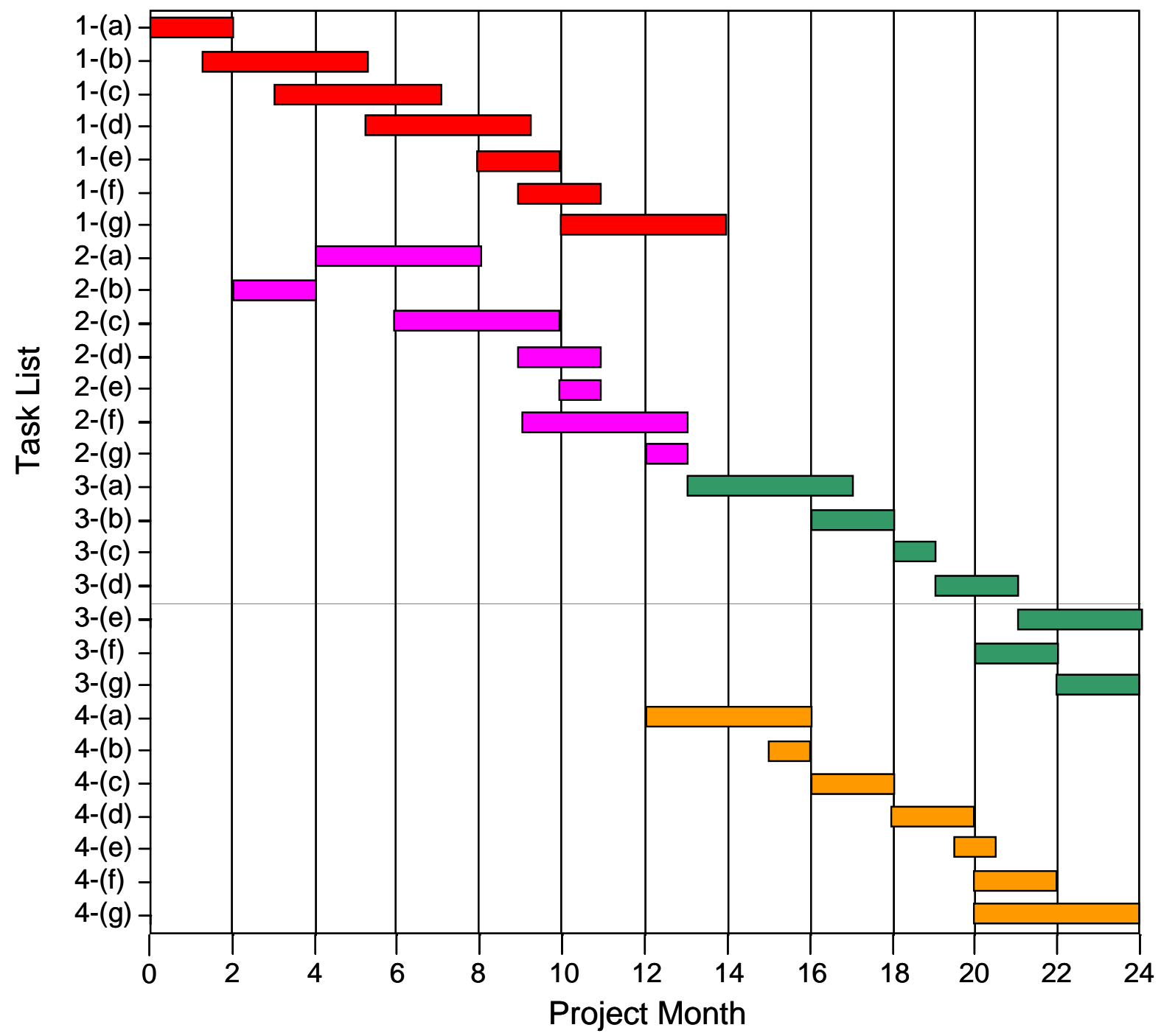

Figure 31. Gantt chart for the four main task objective (indicated by color) broken down by subtasks (see section IV). 


\section{Facilities/Equipment}

As indicated in the previous section, besides the FLC test bed facility, there is a considerable amount of additional diagnostic equipment available for most elements of the proposed work. There is also a fair amount of compression bank hardware as well as vacuum and data acquisition and control equipment. Several components for the test chambers will be supplied by MSNW at no cost. This includes two $1200 \mathrm{l} / \mathrm{s}$ maglev turbo pumps, residual gas analyzer, and a fast ion gauge and controller. Smaller vacuum items to be furnished by MSNW include several fast puff valves gauges and flanges. MSNW will also provide without cost several pieces of data acquisition and control. This includes a gatable, broadband spectrometer, fast response gauss meter, high resolution LCR meter, and several hundred channels of fast transient digitizers. A properly shielded EMI enclosure will also be provided. The significant items to be purchased for the phase II effort will be primarily material and supplies for the macron launchers and macron ring driver banks. Other supplies and materials that will need to be purchased are detailed in the budget section. A breakdown of the expected cost of these items will also be found in the budget section.

The experimental program outlined here for the phase II study is ambitious. In fact, it will require several unique pieces of equipment and magnetic systems that have been developed at MSNW if it is to be accomplished on budget and in a timely manner. Since there isn't sufficient budget for all of the subsystems required, the plan will therefore rely on MSNW to provide either by direct purchase of items, or with in kind contributions to assure the success of the MLF project. The use of the FLC test bed is clearly such a contribution. As part of that effort, the following items will be provided by MSNW without cost to the project:

Osaka Mag-Lev compound turbo-molecular pump system:

(a) Model / P/N TG1113 MBW-09

Controller Model / P/N TD711/1111-C

\section{Calibrated Neutron and Gamma Detector and supply}

(b) Eljen Technology Model 540-20X20-01

\section{$40 \mathrm{kV}$ macron ring driver bank test module:}

(c) Four $-40 \mathrm{kV}, 17 \mu \mathrm{F}$ pulse power capacitors

(c) Steel structure, aluminum feed plates, top hat insulators

(d) Eight Pulse Technology 45k/50 thyratrons

(e) Four Pulse Power Solutions dual heater/driver unit

(f) 100 meters of RG213 cable

(g) switch housings and associated hardware

Macron Launcher and Merging Chamber 
(a) ten port, $60 \mathrm{~cm}$ diameter, stainless steel vacuum chamber and support hardware

(b) 100 channels of fiber optic position sensing devices

(c) Shielded enclosure and snail traps for full scale macron testing 


\section{Bibliography \& References}

[1]. M. Tuszewski, Nuclear Fusion 282033 (1988).

[2] G. Votroubek, J. Slough, S. Andreason, C. Pihl, "Formation of a Stable Field Reversed Configuration through Merging”, Journal of Fusion Energy, Vol. 27, No. 1-2, pg.123 (2007)

[3] Thio, Y.C.F., Knapp, C.E., Kirkpatrick, R.C. Siemon, R.E., Turchi, P.J., “A physics exploratory experiment on plasma liner formation”, J. of Fusion Energy, Vol. 20, No. 1-2 pg. 1 (2001)

[4] P e t e r J. T u r c h i, "Imploding Liner Compression of Plasma: Concepts and Issues", I E E Transactions on Plas $m$ a $\mathrm{Sc}$ i e n c e, $\mathrm{V} \circ \mathrm{I} .36, \mathrm{~N} 0.1,00093-3813\left(\begin{array}{llll}2 & 0 & 0 & 8\end{array}\right)$.

[5] J. H. Degnan et al, "Implosion of solid liner for compression of field reversed configuration”, IEEE Trans. Plasma Sci. 29, 93 (2001).

[6] Degnan, J.H. Amdahl, D.J. Brown, A. Cavazos, T. Coffey, S.K. Domonkos, M.T. Frese, M.H. Frese, S.D. Gale, D.G. Grabowski, T.C. Intrator, T.P. Kirkpatrick, R.C. Kiuttu, G.F. Lehr, F.M. Letterio, J.D. Parker, J.V. Peterkin, R.E. Roderick, N.F. Ruden, E.L. Siemon, R.E. Sommars, W. Tucker, W. Turchi, P.J. Wurden, G.A., "Experimental and Computational Progress on Liner Implosions for Compression of FRCs", IEEE Transactions on Plasma Science, Vol. 36 Issue 1, pg. 80-91 (2008).

[7] Tidman and Massey, “Electrothermal Light Gas Gun”, IEEE Trans.Magn. Vol 29. No 1. pg 621 (1993).

[8] H. D. Fair, “Electric launch science and technology,” IEEE Trans. Magn., Vol 39, No 1, pg. 11, (2003).

[9] Bondelatov and Ivanov, "Ultrahigh axial acceleration of conducting rings", Sov. Phys. Tech. Phys., Vol 22. No 2, pg 234 (1977).

[10] Barry Marder, “A Coilgun Design Primer”, IEEE Trans.Magn. Vol 29. No 1. pg 701 (1993).

[11] R. Paul Drake, James H. Hammer, Charles W. Hartman, L. John Perkins, and Dimitri D. Ryutov, "Submegajoule liner implosion of a closed field line configuration" Fusion Technology, Vol. 30, pg. 310 (1996)

[12]. J.T. Slough, et al, Phys. Rev. Lett., 692212 (1992).

[13] John Slough, Samuel Andreason, Hiroshi Gota, Chris Pihl, and George Votroubek, “The Pulsed High Density Experiment: Concept, Design, and Initial Results", Journal of Fusion Energy, Vol 25, Nos. 3/4 (2006).

[14] Eugene C. Cnare, "Magnetic Flux Compression by Magnetically Imploded Metallic Foils", Journal of Applied Physics, Vol. 27, No. 10,pg. 3812, (1967)

[15] A.L. Hoffman, J.T. Slough, "FRC Lifetime Scaling Based on Measurements from the Large s Experiment (LSX)", Nuclear Fusion 33, 23 (1993). 
[16] T. Intrator et al, "A high density field reversed configuration (FRC) target for magnetized target fusion: First internal profile measurements of a high density FRC", Physics of Plasmas, 11, 2580 (2004).

[17] John Slough, Arthur Blair, Chris Pihl, and George Votroubek, "Magnetically Accelerated Plasmoid (MAP) Thruster - Initial Results and Future Plans", IEPC paper 2007-16, 30th International Electric Propulsion Conference, Florence, Italy, September 17-20, 2007

[18] Giancarlo Becherini and Bernardo Tellini, "Helicoidal Electromagnetic Field for Coilgun Armature Stabilization”, IEEE Trans. Magn., Vol 39, No 1, pg. 108, (2003)

[19] P.Chapelle, et al. "High-speed imaging and CFD simulations of a deforming liquid metal droplet in an electromagnetic levitation experiment", J. of Mat. Sci., Vol 43, No 9, (2008).

[20] Robert E. Peterkin Jr., Michael H. Frese, and Carl R. Sovinec, "Transport of Magnetic Flux in an Arbitrary Coordinate ALE Code", J. Comp. Phys. 140, 148 (1998)

[21] Degnan, J.H. Amdahl, D.J. Brown, A. Cavazos, T. Coffey, S.K. Domonkos, M.T. Frese, M.H. Frese, S.D. Gale, D.G. Grabowski, T.C. Intrator, T.P. Kirkpatrick, R.C. Kiuttu, G.F. Lehr, F.M. Letterio, J.D. Parker, J.V. Peterkin, R.E. Roderick, N.F. Ruden, E.L. Siemon, R.E. Sommars, W. Tucker, W. Turchi, P.J. Wurden, G.A., "Experimental and Computational Progress on Liner Implosions for Compression of FRCs", IEEE Transactions on Plasma Science, Vol. 36 Issue 1, pg. 80-91 (2008). 


\title{
Macron Formed Liner as a Practical Method for Enabling Magneto-Inertial Fusion
}

\author{
David Kirtley $\cdot$ John Slough
}

Published online: 17 July 2010

(C) Springer Science+Business Media, LLC 2010

\begin{abstract}
To take advantage of the smaller scale, higher density regime of MIF an efficient and repeatable method for achieving the compressional heating required to reach fusion gain conditions is needed. The macro-particle (macron) formed liner compression of the field reversed configuration (FRC) provides such a method. The approach to be described employs an assemblage of small, gram scale, macrons to form a more massive liner that both radially and axially compresses and heats the FRC plasmoid to fusion conditions. The large liner energy (several MJ) required to compress the FRC is carried in the kinetic energy of the full array of macrons. The much smaller energy required for each individual macron is obtained by accelerating the macron to $\sim 3 \mathrm{~km} / \mathrm{s}$ which can be accomplished remotely using conventional inductive techniques. 3D numerical calculations demonstrate that macron convergence can form a coherent liner provided minimum velocity and timing accuracy is met. Experimental results have demonstrated that a cylindrical or spherical macron can be accelerated to velocity within $2 \mathrm{~m} / \mathrm{s}$ and timing less than 1 microsecond. Initial testing of a 6-stage launcher yielded $280 \mathrm{~m} / \mathrm{s}$ at a final coupling efficiency of greater than $40 \%$.
\end{abstract}

Keywords Magneto-inertial fusion . Magnetic liner compression · FRC

D. Kirtley · J. Slough $(\bowtie)$

MSNW LLC, 8551 154th Avenue NE, Redmond,

WA 98052, USA

e-mail: sloughj@comcast.net

\section{Introduction and Methodology}

To take advantage of the smaller scale, higher density regime of magneto-inertial fusion (MIF) an efficient method for achieving the compressional heating required to reach fusion gain conditions must be found. The method needs to be simple and capable of repetitive operation. The macroparticle (macron) formed liner compression of the FRC is such a method, and uniquely addresses the major challenges facing magneto-inertial fusion. Specifically, it embodies a compression scheme that can very efficiently and repetitively generate the kinetic liner energy required to reach fusion gain. It provides for both the target plasma and liner energy to be generated remote from the reactor vessel. This is critical as the reactor environment is likely to be incompatible with the specialized pulse power equipment employed in conventional liner approaches. The timescale for forming and accelerating both the FRC and liner can be much longer than the time that the energy is thermalized in the implosion. This avoids the need for the very high voltages required to produce multi-megampere compression currents. In fact the switching requirement for the macron formed liner (MFL) is well within the range of currently available solid state devices. The time and cost for testing the concept is negligible when compared to alternative approaches. The process required for target FRC formation is already routinely produced at the MSNW laboratory [1].

The key to attaining a workable pulsed fusion reactor is achieving the required standoff for the liner driver as is illustrated in Figs. 1 and 2. The target plasma is assembled by producing two FRCs remote from the burn chamber, and translating them into the center where they merge and form a stable target FRC. The merged FRC must have a decay time long compared to the FRC assembly and 


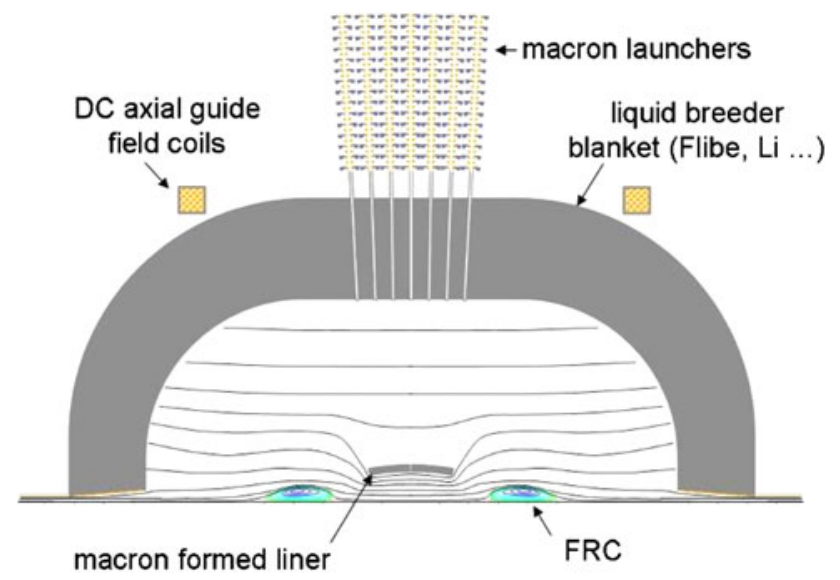

Fig. 1 Reactor illustration based on the macron formed liner $(M F L)$ compression of the FRC ( $R-Z$ plane). Scale of the FRC and liner has been enlarged for purpose of illustration

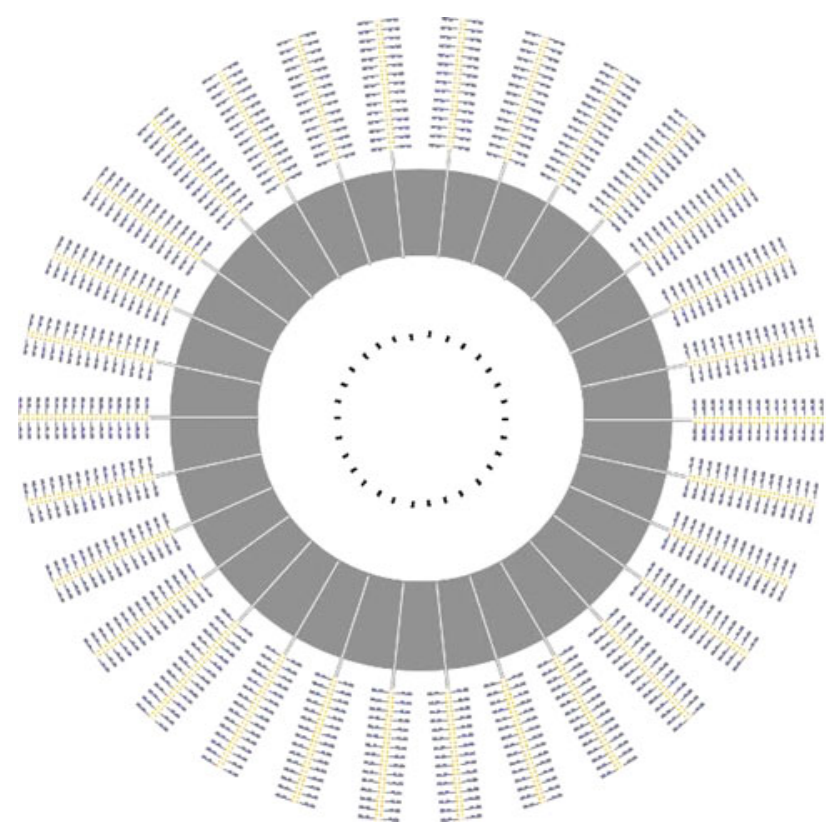

Fig. 2 Midplane cross sectional view of reactor based MFL. The macron size was increased and reactor scale reduced for illustration

compression time-but not the liner formation time which can be initiated much earlier. This aspect of the conceptthe FRC formation, acceleration and merging process-has already been successfully demonstrated in the inductive plasma accelerator (IPA) experiments at MSNW [2]. In the reactor application a small $(\sim 0.1 \mathrm{~T})$ guide field maintains the FRC against radial expansion as illustrated in Fig. 1.

The metallic liner is also produced by merging as well, however, in this case by a cluster of small masses (macrons) that are injected into the reactor by an array of launchers, also situated outside the reactor vessel. In this way the energy required to compress the FRC can be delivered on a timescale that is much slower than the FRC compression, and produced by a source that is highly efficient as well as broadly distributed spatially. Most importantly, the liner formation system can be located outside the reactor in a manner compatible with long-term repetitive operation.

The macron launchers are initiated in such a manner that a large array of small metallic masses arrive at the central section of the reactor (see Fig. 2) and converge to form a contiguous liner at smaller radius $\left(r_{\mathrm{L}} \sim 0.1 \mathrm{~m}\right)$. The FRC is then introduced within the liner by merging two translated FRCs.

The shape of the macron will be considered later, but for this discussion its radial size and mass are the relevant parameters. The scale and number of macron launchers required for the liner can be readily estimated by what was achieved in the MTF liner experiments performed at AFRL [3]. A cylindrical aluminum shell liner of roughly $300 \mathrm{~g}$ mass was imploded using the 4.5 MJ Shiva Star capacitor bank. The $5 \mathrm{~cm}$ radius liner was compressed with an axial current of $12 \mathrm{MA}$ at $84 \mathrm{kV}$ and produced a final liner kinetic energy of $\sim 1 \mathrm{MJ}$ in $22 \mu \mathrm{s}$ [4]. Employing the macron launcher it should be possible to exceed this liner energy by a factor of three for a similar stored energy. For example, the target parameters for each launcher are a macron mass of $4 \mathrm{~g}$ accelerated to a velocity of $3 \mathrm{~km} / \mathrm{s}$ $(3 \mathrm{~mm} / \mu \mathrm{s})$. The kinetic energy of each macron would thus be $\sim 20 \mathrm{~kJ}$. For a $1 \mathrm{~cm}$ radius macron, the 30 macron launchers depicted in Fig. 1 would converge to make contact at a radius of roughly $10 \mathrm{~cm}$ and be well merged at $5 \mathrm{~cm}$. Five such rings arranged axially would initially form a liner of length comparable to the AFRL liner, and have roughly twice the mass $(0.6 \mathrm{~kg})$. The liner kinetic energy, however, would total $3 \mathrm{MJ}$. And it would compress from $5 \mathrm{~cm}$ radius to the minimum $(r<2 \mathrm{~mm})$ in less time ( $\sim 15 \mu \mathrm{s})$. In addition to a higher kinetic energy, the macron liner can be made to converge axially thereby increasing the compressional energy even further. This is an significant advantage as the FRC equilibrium length contracts axially $\left(1-r^{2 / 5}\right)$ as it is compressed. With a fixed liner length, the efficiency of compression is greatly diminished by this effect. Without electrical contacts, the experimental apparatus and vacuum system can also be greatly simplified. Difficulties with the post implosion vacuum integrity are also much easier to avoid. A unique benefit of macron assembled liners is the ability to impart a specified amount of rotation. The rotating inner surface will be stabilized to instabilities during the final compression stages. The amount of rotation to be added to the liner must balance stability, shear, and overall energy efficiency.

It should be noted that the small aperture in the chamber wall required for macron introduction is quite small $\left(\sim 3 \mathrm{~cm}^{2}\right)$, so that even with several hundred such holes, the portion of reactor wall area exposed would be less than 
a small fraction of a percent of the total area $(<0.1 \%$ of a $3 \mathrm{~m}$ diameter chamber). The time from launch to the arrival of detritus from the liner implosion will be several milliseconds providing sufficient time to aperture the small ports closed. The neutron fluence to the launcher structure is also significantly reduced by being completely outside the blanket with only a small acceptance angle for exposure due to the long connection tube length through the blanket. The distributed launcher array greatly reduces the demand on the power delivery systems. With a constant acceleration of the macron to $3 \mathrm{~km} / \mathrm{s}$ over a distance of $3 \mathrm{~m}$, the liner energy input can occur over a time span as long as $2 \mathrm{~ms}$ - two orders of magnitude slower than the conventional $\mathrm{Z}$ pinch method. As will be seen, the energy input from each macron launcher is low enough and slow enough that it can be provided with commercial solid state electronics.

There are three key issues that must be solved for the macron formed liner concept to be validated. First, the timing accuracy and velocity spread must be able to deliver the required liner behavior. Secondly, the dynamical behavior of the liner during compression must be understood, specifically how it is influenced by non-uniformities or rotation. Finally, the optimal projectile geometry, trajectory, and number must be fully characterized. The answer to these three questions from the initial results of the 3D modeling indicates that a suitable liner can be formed. The modeling results are discussed in more detail in section "Modeling Results". The most critical issue is to demonstrate the ability to produce the desired macron kinetic behavior in a consistent and repeatable manner. It is clear from the considerations above; the launcher must be simple, reliable and robust. It is believed that the prototype design possesses all these features. Initial testing of the launcher is described in section "Experimental Results".

There is a wide array of possible methods to achieve the desired velocities and masses. The velocity requirement is rather modest compared to that attained by a large range of projectile acceleration methods. There are both electromagnetic (e.g. rail guns) and gas dynamic approaches (e.g. light gas guns) that have achieved velocities far in excess of the $3 \mathrm{~km} / \mathrm{s}$ target velocity. Pulsed Inductive acceleration employing a series of sequenced coils as illustrated in Fig. 3 turns out to be the preferred method for several reasons. For methods that rely on physical motion for initiation such as light gas guns and pistons, it is highly unlikely that an array of such devices that could achieve the timing jitter required for the liner assembly. As a crude estimate of what timing would be required, consider the arrival of a $2 \mathrm{~cm}$ long macron at the point of contact. If a position accuracy of $2 \mathrm{~mm}$ was desired, the firing time jitter at a macron closing speed of $3 \mathrm{~km} / \mathrm{s}$ would be $\sim 0.6 \mu \mathrm{s}$. While larger variations may prove to be

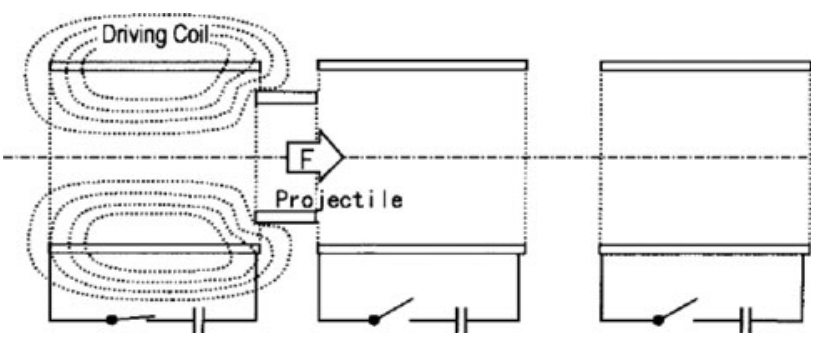

Fig. 3 Pulsed inductive acceleration of a cylindrical macron

acceptable, it will certainly be of this order. While this is an easily achieved jitter for EM launch techniques, achieving velocity control as well eliminates techniques such as rail guns where rail contact and other issues limit the accuracy that can be achieved in terminal velocity. With the inductive accelerator, the motion of the macron is controlled by the propagating magnetic wave which can be made identical in each accelerator. Variation in macron response to acceleration due to variations in size or mass are largely self-correcting due to the axial gradient in the force imparted by the propagating field. If the macron moves out ahead of the wave the axial driving force experienced by the macron diminishes bringing the macron back into alignment. The same effect corrects for a macrons that fall behind requiring greater force.

The force on the projectile can be easily is derived from Lens' law with an equation of motion that depends on the coil-projectile geometry and currents:

$F(z, t)=I_{\mathrm{c}}(t) \cdot I_{\mathrm{p}}(t) \frac{\mathrm{d} M_{\mathrm{cp}}}{\mathrm{d} z}$,

where $M_{\mathrm{cp}}$ is the mutual coupling between coil and projectile. Integrating the above equation along the axis of motion yields the work done on the projectile and thus the final kinetic energy of the projectile:

$\frac{1}{2} m_{\mathrm{p}} v_{\mathrm{p}}^{2}=I_{\mathrm{c}} I_{\mathrm{p}} \int_{0}^{\infty} M_{\mathrm{cp}} \mathrm{d} z=\frac{I^{2}}{2} M_{\mathrm{cp} 0}$

where $M_{\mathrm{cp} 0}$ is the value of the average mutual coupling to the array of coils experienced by the projectile along the length of the accelerator. It was assumed during the projectile acceleration that the current in the coil is oscillatory, and that the macron image currents completely screen the solenoidal coil fields $\left(I_{\mathrm{p}}=I_{\mathrm{c}} \equiv I\right)$. To maximize the efficiency of the driver, the drive coil circuits execute a full period before the circuit is opened. This allows any energy not transferred into macron motion to be returned to the capacitor for future use. The direction of the axial force is independent of field direction so that the axial force is continued with current reversal. By using an oscillatory current the magnetic field is kept from penetrating the 
macron and reducing the accelerating magnetic gradient. The polarity of each coil is reversed and timed so that the field is in alignment with the field produced during the previous coil's second half cycle. The stability of the macron is also much improved with this acceleration method as the direction of the dipole moment alternates as the macron moves from coil to coil preventing a possible tilt instability from growing. A multipole (quadrupole) guide field can also be applied to assure alignment of the macron and to maintain clearance with the accelerator wall.

\section{Modeling Results}

The 3-D colliding macron liner was simulated using ANSYS Multiphysics which has the capability of calculating the behavior of the colliding aluminum macrons well into the non-linear plastic deformation regime. The initial calculations were performed for macrons comprised of hollow aluminum spheres, $6.7 \mathrm{~g}$ in mass which were launched radially inward at $3 \mathrm{~km} / \mathrm{s}$. The spheres were observed to collide, deform, and finally form a complete, uniform liner. The entire process occurred with little diminution in the radial velocity. For the target velocity of $3 \mathrm{~km} / \mathrm{s}$ the macron merging and compression is sub-sonic. The simulation was 3 dimensional and both stabilizing rotation (initiated with small non-radial velocity component) and 3-D compression (using 2-3 arrays of macron liners) were modeled. Finally, a complete treatment of the transient structural physics of the macron collision and integration was performed, including full plastic deformation stress-strain relationships, detailed surface roughness, and interaction studies.

Studies of liner uniformity and stability showed that the readily compressive nature of the hollow macrons themselves allows for a fairly large tolerance in initial velocity and timing, and still yield a uniform liner compression. It was found that initial positioning of up to one half macron radii ( $25 \%$ of total scale) is tolerable. For a $3 \mathrm{~m}$ diameter chamber this corresponds to an initial velocity and timing accuracy of $15 \mathrm{~m} / \mathrm{s}$ and $2.5 \mu \mathrm{s}$. Surprisingly, it was found that even a fully missing macron is compensated for during the compression process. It was observed that greater than 95\% of initial kinetic energy is maintained during maximum compression. A key advantage to this method of liner formation is the ability to aim the macrons to converge axially matching the FRC contraction and increasing the compressional energy on target. The macrons can also be aimed slightly off the axis of symmetry in order to provide rotational stability to the liner. The calculation shown in Fig. 4 illustrates the liner formation process employing 20 hollow aluminum macrons. In addition to the timing and

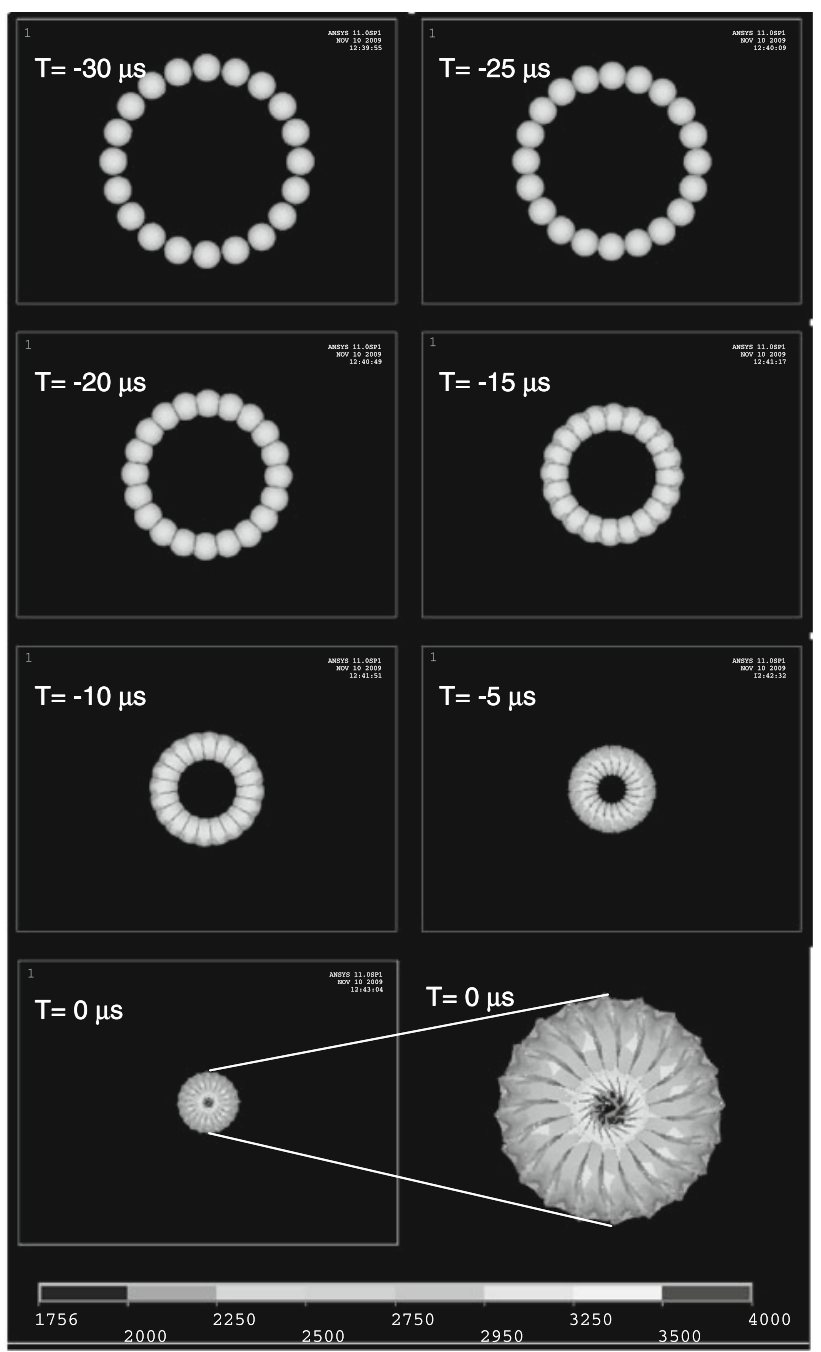

Fig. 4 Macron velocity profiles during formation of a 1.5 MJ liner formed employing 20 hollow spherical aluminum macrons moving radially inward at $3 \mathrm{~km} / \mathrm{s}$ with the center of mass $5 \mathrm{~mm}$ off-axis to impart angular momentum to the liner for stabilization

velocity perturbations, the modeling showed that the high compressibility of the macrons allowed for significant rotation and axial variations to be added to the liner while still maintain maximum radial velocity.

Several aspects of the macron liner dynamics still need to be resolved. A significant issue concerns the behavior of the inner surface of the merged macron liner during field compression and stagnation. With macrons there is the possibility of a more irregular inner surface which could be prone to spiking. There are two effects that should ameliorate this tendency. As has been mentioned, it is trivial to add a large rotational motion to the inner wall by a coherent non-radial displacement of the macron initial trajectory. As can be seen in Fig. 4 this causes a rapid winding up and smearing of the inner boundary. The rotational motion is also stabilizing to the Rayleigh-Taylor 
modes that are the primary cause of the spiking. With a compressed megagauss magnetic field, there will also be large wall currents that would lead to rapid melting of small protuberances on the inner wall.

It should be possible to resolve the full macron liner behavior during magnetic field compression and stagnation with the same ANSYS Multiphysics code used in the initial simulations. To achieve this result, future code work will incorporate an axial magnetic field along with modifications to include material phase change and a temperature dependent resistivity. It will also require a finer mesh than was used in these calculations. With these improvements it should be possible to establish the dynamic stability and compressive efficiency of the MFL.

\section{Experimental Results}

A six-stage macron launcher was constructed in order to determine the efficiency and accuracy of gram-scale pulsed-inductive launchers. The initial launcher was constructed with six separate coil stages. Each $1.3 \mathrm{~cm}$ long stage consisted of a 5-turn $1.9 \mathrm{~cm}$ diameter coil made from 13 gauge Litz wire each potted in a low-viscosity epoxy. Each coil was driven with a $600 \mathrm{uF}$ electrolytic capacitor bank switched with six paralleled 1,700 V Isolated Gate Bipolar Transistor (IGBT) switches producing a peak current of $24 \mathrm{kA}$ at $1 \mathrm{kV}$ resulting in an axial magnetic of up to 25 Tesla with a cylindrical macron. Between the coils a high-intensity, $660 \mathrm{~nm}$ fiber transmitter/receiver was positioned for velocimetry measurements (see Fig. 5). The initial launcher is shown in Fig. 5.

The six stage-launcher successfully demonstrated the pulsed-inductive acceleration of cylindrical macrons with masses ranging from 1 to $4 \mathrm{~g}$. Variable aspect ratio cylinders and spheres were also examined. The six stage

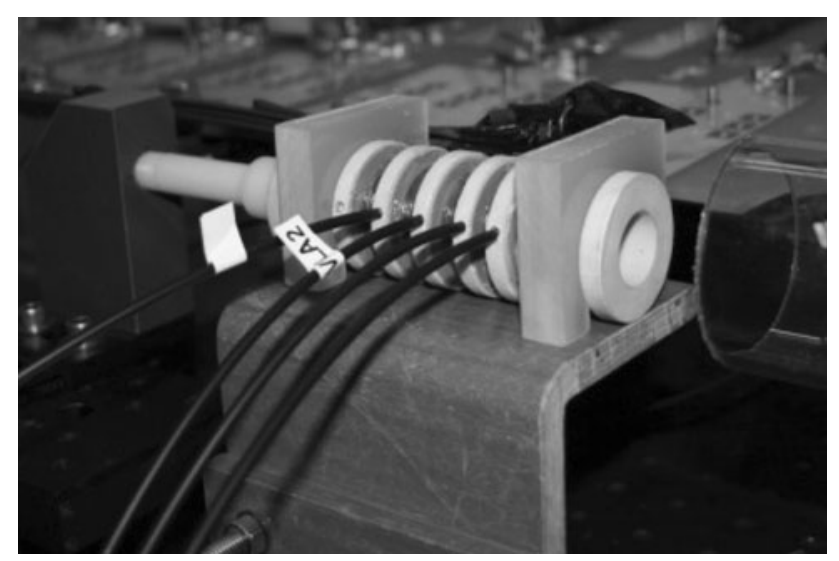

Fig. 56 stage macron launcher launcher demonstrated a peak velocity of $280 \mathrm{~m} / \mathrm{s}$ and validated performance expectations for the limited energies tested. Several important characteristics were exhibited. The jitter from sequential launches with different macrons was observed to be near the resolution of the digitizer at a fraction of a microsecond. The macron arrival times varied by less than $1 \mu$ s corresponding to a velocity accuracy of $2 \mathrm{~m} / \mathrm{s}$. Additionally, velocity could be pre-programmed to less than $5 \mathrm{~m} / \mathrm{s}$. An initial position study was performed as it was found that maintaining the initial position of the macron was important in minimizing the jitter as well as the final macron velocity. This is primarily due to the accelerator coil inefficiency at low macron velocities where the magnetic field oscillations were not synchronized well with the macron passage. A key parameter in coupling efficiency is the mutual inductance between macron and the driver coil [see (2)]. As this quantity is difficult to calculate accurately, it was found the most straight forward (as well as most appropriate) way was to simply measure it. This was done for both the cylindrical and spherical macrons with the results shown in Fig. 6. The coupling was higher for the cylindrical macron, but the spherical macron produced a better force gradient for position and velocity control. As mentioned, it is difficult to properly drive the macron during the startup phase. The energy coupling efficiency, $\eta_{\mathrm{e}}$, is defined as the ratio of the kinetic energy acquired by the macron to the coil magnetic energy. As indicated in Fig. 7, the energy coupling steadily improves for later stages at higher macron velocity where $\eta_{\mathrm{e}}$ approaches the theoretical maximum of $45 \%$ inferred by (2) and the measured mutual inductance for the prototype coils and cylindrical macrons. Higher efficiencies are achievable with larger coils. Different macron shapes will also be tested such as a bullet shaped macron that combines the advantages of both the cylinder and spherical macrons

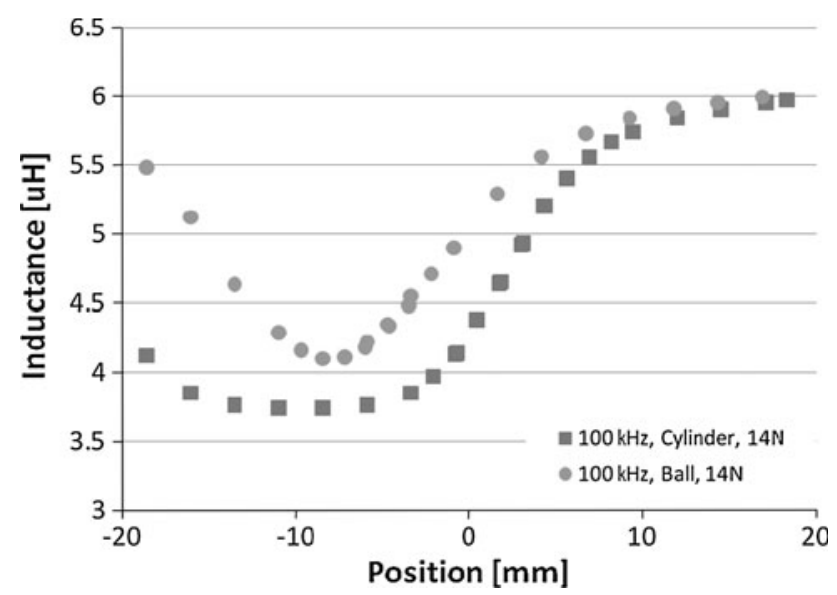

Fig. 6 Coil and macron total inductance for cylindrical and spherical macrons, measured as a function of position relative to the coil center 


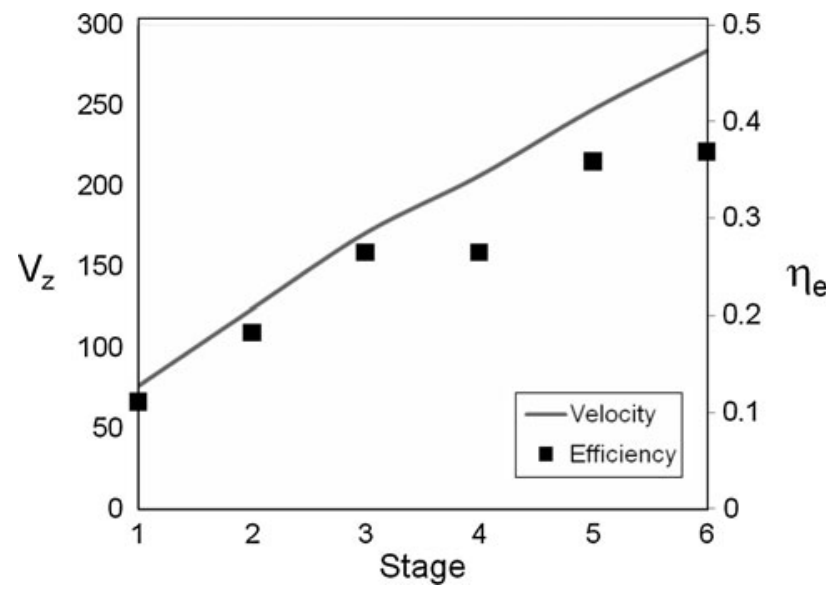

Fig. 7 Macron velocity and energy coupling efficiency at each stage

considered so far. Future work will increase the number of stages to bring the macron velocity closer to that desired for the reactor application.

\section{References}

1. G. Votroubek, J. Slough, Plasma Liner Compression of a Field Reversed Configuration. (this issue)

2. G. Votroubek, J. Slough, S. Andreason, C. Pihl, J. Fusion Energ. 27(1-2), 123 (2007)

3. J.H. Degnan et al., IEEE Trans. Plasma Sci. 29, 93 (2001)

4. J.H. Degnan et al., IEEE Trans. Plasma Sci. 36(1), 80-91 (2008) 